\title{
Dual Stage Bayesian Network with Dual-Tree Complex Wavelet Transformation for Image Denoising
}

\author{
P.Venkata Lavanya*, C.Venkata Narasimhulu** and K.Satya Prasad*** \\ *Research Scholar, Department of ECE, Jawaharlal Nehru Technological University, Kakinada, Kakinada, Andhra Pradesh, India \\ **Department of ECE, Geethanjali College of Engineering and Technology, Hyderabad, Telangana, India \\ ***Vignan's Foundation for Science, Technology and Research, Guntur, Andhra Pradesh, India \\ *Corresponding Author: pvlavanya999@gmail.com
}

\begin{abstract}
Image denoising always plays a vital role in various engineering bids. Moreover, in image processing technology, image denoising statistics is persisted as a substantial dispute. Over the past decades, certain denoising methods have reached incredible accomplishments. Since there is no much contribution on image denoising considering multimodal and heterogeneous images, this paper motivates us to extend it with the aid of intelligent approach. Dual-tree Complex Wavelet Transform (DT-CWT) is exploited for image transformation for which the wavelet coefficients are estimated using Bayesian Regularization (BR). To ensure the denoising performance for heterogeneous images, the statistical and wavelet features are extracted. Subsequently, the image characteristics are combined with noise spectrum to develop BR model, which estimates the wavelet coefficients for effective denoising. Hence, the proposed denoising algorithm exploits two stages of BR. The first stage predicts the image type, whereas the second stage estimates appropriate wavelet coefficients to DT-CWT for denoising. As a main contribution, the filter coefficients of DT-CWT are optimized by Genetic Algorithm (GA). The performance of the proposed model is analysed in terms of Peak Signal to Noise Ratio (PSNR), Second derivative Measure of Enhancement (SDME), Structural Similarity (SSIM), Mean Squared Error (MSE), Root Mean Square Error (RMSE), Mean Absolute Error (MAE), Pearson Coefficient (PC), and Symmetric Mean Absolute Percentage Error (SMAPE), respectively. The proposed model is compared to the conventional models, and the significance of the developed model is clearly described. From the analysis, it is observed that the PSNR of the developed model is $69.97 \%, 5.85 \%, 76.91 \%, 33.38 \%, 46.40 \%$, and $46.44 \%$ better than 2D SMCWT, DT-CWT, DT-CDWT, DT-RDWT, W-ST, and W-HT, respectively. Similarly, for SSIM measure, the proposed model has great deviation over conventional methods, and the model is $19.17 \%, 83.66 \%, 24.65 \%, 72.99 \%$, and $73.15 \%$ better than DT-CWT, DT-CDWT, DT-RDWT, W-ST, and W-HT, respectively.
\end{abstract}

Keywords: Bayesian Regularization; DT-CWT; Genetic Algorithm.; Image Denoising; Filter coefficient.

\section{INTRODUCTION}

Image noise removal is a necessary pre-processing function in diverse optical engineering applications (Liu \& Fang, 2015; Wang \& Kai Fu, 2010; Nasri \& Nezamabadi-pour, 2009; Jin, 2013; Liu et al., 2009; AM Wagh \& Todmal, 2015; Sable \& Jondhale, 2010). The multiple noises occurred from the image acquisition, and transmission process destroys the image quality. In the domain of image processing, image denoising has been persisted as a significant issue (Guo et al., 2017; Hou et al., 2011; Wang et al., 2013; Ghorai, 2013; Xin \& Jiangtao, 2011). As a challenge to this issue, numerous researchers have developed several noise reduction techniques for the removal of noise from the image. The development of new sensors and the improvement of image denoising models have eventually brought the remote sensing community to consider the use of satellite images acquired under heterogeneous conditions (Wei et al., 2017; Maoguo et al., 2016; Zhun-ga et al., 2017; Luigi et al., 2017). This has led to methods based on heterogeneous sources of data (Wei et al., 2017; Puzhao et al., 2016; Zhun-ga et al., 2017; G. Mercier et al., 2008; Jorge et al., 2015; Zhunga et al., 2018), also referred to as multi-source (Maoguo et al., 2016; Devis et al., 2016), multi-modal 
(Redha et al., 2018), multi-sensor or cross-sensor (Bard et al., 2009; Michele et al., 2015; Redha et al., 2018), and information unbalanced data (Linzhi et al., 2017). Among those techniques, more conventional algorithms execute image denoising process in the pixel domain. Over the past decades, some denoising techniques based on transform domain have revealed incredible achievements (Rabbani, 2009; Shang et al., 2012; Yang et al., 2012; Wong et al., 2011; Luo \& Zhu, 2012; Decker et al., 2010). In fact, the transform-based denoising characteristically approximates the true signal by a linear grouping of some basic features (Liu et al., 2008), which specifies the signal in the form of transform domain. Thus this technique transfers the true signal energy by conserving some transform coefficients with high magnitude and destroys the uncertainty due to noise, leading to estimate the true signal in an effective manner. Moreover, the properties of transform, as well as true signal, provide the sparsity of overall representation.

In fact, a number of standard models are there for denoising the noisy image, which has the capability of performing distinct filtering process that minimizes the level of noise. However, the image comes under the blur condition or under over smoothed since it loses its edges. Noise minimization is utilized to eliminate the noise even without losing any details present in the images. Some of the spatial filters, such as mean as well as median, are utilized to eliminate the noise from the corresponding image. However, a drawback of the spatial filter is that while smoothing the image, the edges become blurred. Thus, the wavelet transform is very efficient to preserve the image's edges. It is considered as one of the powerful tools of signal processing because of its multi-resolution possibilities. Wavelets also produce the effective performance in denoising the image with its precious properties like sparsity as well as multi-resolution structure. Moreover, wavelet transform (Li et al., 2016) has already shown its effectiveness in removal of noise, also minimizes the complexity of the computations, and facilitates best noise minimization performance.

The wavelet transform achieves effective sparsity for localized details including edges and singularities. Basically, those details are typically very high in natural images and also convey the particular portion the data embedded in, and the wavelet transform is represented as the distinct application for denoising of image (Wang et al., 2010). More advanced denoising models are developed based on wavelet transforms (Beck \& Teboulle, 2009; Luisier et al., 2007; Pizurica \& Philips, 2006; Portilla et al., 2003; Yan et al., 2008). For some particular natural images, the wavelet coefficients comprise small magnitudes, but present some large magnitude also, which is considered as the vital highfrequency features of image including edges. Between all wavelet coefficients, the white noise is distributed evenly, but while eliminating small wavelet coefficients, minimizing the noise energy at the time of preservation. The problem of image noise suppression remains an open challenge, especially in situations where the images are acquired under poor conditions where the noise level is very high. To denoise an image, wavelet transform is adopted because it gives coefficients with respect to the functions. Once the coefficients are obtained, they can be used for any purpose. With that coefficience, one can reconstruct, denoise, compress the image, etc.

This paper contributes an intelligent image denoising model. The proposed denoising algorithm exploits two stages of BR. The first stage categorizes the image types, whereas the second stage estimates appropriate wavelet coefficients to DT-CWT for denoising. The paper intends to exploit BR since it explores advantages like easier implementation, good result obtainment in most of the cases, and also it necessitates a smaller amount of training data to estimate parameters (Aggarwal et al., 2005). Further, the filter coefficient parameter of DT-CWT is optimally selected using Genetic Algorithm (GA), which is chosen because it can find fit solutions in much less time. Moreover, it is intrinsically parallel and gives chances for getting more optimal solutions. In addition, DT-CWT parameters solve the problems of shift variance and low directional selectivity in two and higher dimensions found with the commonly used Discrete Wavelet Transform. Since the Bayesian Network has been used for both image classification and image denoising, the process is termed as Dual-stage network. The proposed model is compared to conventional methods like 2D Scale Mixing CWT (2D SMCWT), DT-CWT, Dual-Tree Complex DWT (DT-CDWT), Dual-tree Real DWT (DT-RDWT), Wavelet Soft Thresholding (W-ST), and Wavelet Hard Thresholding (W-HT). The rest of the paper is organized as follows: Section II reviews the literature work. Section III describes the developed image classification model. Section IV explains the weight optimization based image denoising strategy. Section V discusses the results obtained, and Section VI concludes the paper. 


\section{LITERATURE REVIEW}

In 2017, Guo et al. have presented the image denoising model, which was on the basis of 'Stationary Wavelet Transform (SWT)' along light noise. SWT based denoising procedure was offered after the analysis of light noise. With the use of this denoise algorithm, the developed model was established conforming to granting accurate DIC measurements even in the light noise scenario. Further, the developed method was compared with the existing models, and the real-time experimentation was also carried out to ensure the performance of the model. From the results, it was evident that the developed denoising model could be applied for the full-field strain measurement in light interference with more accuracy and stability, respectively.

In 2016, Li et al. have developed a 'Wavelet-based Contour let transform (WBCT)' model to achieve an Adaptive Optics image denoising. This model was implemented via 'Bayes Shrink' theory for the assessment of the threshold. Then they also contributed to the enhancement of the adaptive model for threshold choosing by which they have attained optimal threshold. Here, the denoising of image was achieved by choosing the adaptive optimal threshold by using the WBCT transform coefficients of varied decomposition scale and directions. The algorithm was evaluated with the aid of 'DTCWT-BayesShrink algorithm', DWT-NA Bayes Shrink algorithm', and 'CbATD algorithm'. The developed model was examined using real AO images and simulated images. From the analysis, they have attained better performance in terms of 'peak signal-to-noise ratio (PSNR)' and visual quality as well.

In 2014, Remenyi et al. have developed an image denoising model, which was on the basis of '2D scale-mixing complex-valued wavelet transform'. To attain this, they have used both unitary (minimal) and maximal (redundant) versions. By this, they have established the covariance structure of a noise, namely, white noise in wavelet domain. The model assessment was achieved through empirical Bayesian modalities that include the preserved versions of the complex-valued wavelet coefficients. Hence, the new procedure has displayed the perfect quantitative performance as well as visual performance through simulation.

In 2015, Jesus et al. have established a model for high resolution small animal 3D PET data. This has been aimed for the minimization of noise and for providing the detailed pervasiveness. This was on the basis of assessment of the non-subsampled Haar wavelet coefficients with the aid of linear estimator. The model was applied to the images like volumetric images, which were reconstructed without alteration influences (reconstruction). The simulation results have shown the pervasiveness of the developed model, and also the method has ultimately minimized the noise presented in the image.

In 2014, Sun et al. have presented a novel image denoising model with the use of linear Bayesian 'Maximum a Posteriori (MAP)' assessment on the basis of sparse demonstration classic. In the preliminary step, the probability dissemination was constructed in representation vector. They have also constructed the 'linear Bayesian MAP estimator' for obtaining the probabilistic observation. This was adopted for solving the problem, named inverse problem. Moreover, they have also attained the solution for closed-form solution and hence, they have achieved better image denoising strategy. They have also classified them to various sub-groups with their patterns and performed the training process of various dictionaries with the aid of 'K-SVD algorithm.' The denoised image was acquired by smearing the denoising to each subgroups, which was on the basis of estimator and also by doing mean of the two outputs. The proposed model was compared to the existing models. The simulation results have shown the competitive performance of the developed denoising model in terms of both visual quality and PSNR value.

In 2016, Song et al. have developed a deblurring model, which was based on 'Gradient Histogram Preserving (GHP)'. In the methods used for the development, they were parameterized with Hyper-Laplacian dissemination. With the consideration of blurring complexity, a Bayesian model named Gaussian processes reversion was used for assessing the parameters of the histogram. The experimentation was carried out for the developed model, and the results have viewed the efficiency of the parameter assessment. Moreover, the quality of the image was highly enhanced, and the restoration of the image was well performed. 
In 2015, Naimi et al. have developed a denoising model, which was on the basis of DTCWT with wiener filtering model. The experimentation was carried out for the proposed image and from the results, it was proved that the denoised images with DTCWT have more balancing power among smoothness as well as the accuracy, whereas the remaining models like SWT (Stationary Wavelet Transform) have less accuracy. They have also utilized the SSIM (Structural Similarity Index Measure) with PSNR as well as SSIM map for the assessment of the image quality.

In 2013, Zhang et al. have established the model of the combination of 2D discrete wavelet transform and bilateral denoising. Initially, they have adopted the wavelet transform for doing the image decomposition in which the component of low frequency persisted unaffected. Later, the image is reconstructed by treating the bilateral filtering. The developed model was compared to the existing models and from the results, it was proved that the combinations of wavelet transform and bilateral filtering could attain the betterments in retaining the details of the image. Moreover, it was also achieved the efficient visual effect. The model was identified as the efficient one while comparing with the bilateral filtering alone and wavelet transform alone.

One of the primary challenges in the field of image processing is image denoising, where the underlying goal is to estimate the original image by suppressing noise from a noise-contaminated version of the image. Image noise may be caused by diverse intrinsic and extrinsic conditions, which are often not possible to avoid in practical situations. Several researchers are undoing research on image denoising concept, where they have proposed diverse algorithms for the purpose of image denoising. The detailed review papers on image denoising by wavelet transform is shown in Table 1. The different wavelet transforms adopted in the literature include Stationary wavelet transform (Guo et al., 2017), Contourlet transform (Li et al., 2016), Complex wavelet transform (Remenyi et al., 2014), Haar wavelet transform (Jesus et al., 2015), Bayesian MAP estimation (Sun et al., 2014), Gradient histogram (Song et al., 2016), DT-CWT (Naimi et al., 2015), and 2D-DWT (Zhang, 2013). Image denoising using Stationary wavelet transform (Guo et al., 2017) provides high accuracy and high stability; yet there is a presence of very large redundancy, and the computational complexity is increased. Next, better restoration quality and high PSNR is attained by the Contour let transform ( $\mathrm{Li}$ et al., 2016), whereas it requires more calculation time and its basic images are localized in the frequency domain. Moreover, the Complex wavelet transform (Remenyi et al., 2014) offers excellent quantitative and visual performance and it is conceptually simple and practically efficient algorithm, but it fails to perform the threshold limit selection based on statistical models of wavelet coefficients. The noise of the image is drastically reduced by the Haar wavelet transform (Jesus et al., 2015); yet, it is continuous and therefore non-differentiable and cannot compress the energy of the original signal into a few high-energy values. The Bayesian MAP estimation (Sun et al., 2014) is an effective method to remove noise that is possible to obtain sharp edges and clear textures and high quality. However, the information is theoretically infeasible, and the process is not automatic and computationally infeasible. In addition, the restoration of image texture is easy in gradient histogram (Song et al., 2016), and it exhibits improved image quality, but the robustness and the detection speed are very low. Moreover, DT-CWT (Naimi et al., 2015) provides high efficiency in denoising, less redundant and easy computation, whereas it takes a long time as the process is too long. Furthermore, 2D-DWT (Zhang, 2013) can retain all the required details from the image and suppress the image noise. However, the complexity is high and it is theoretically difficult to understand and interpret the results.

In recent years, wavelet transform has attracted significant attention in scientific research and engineering applications since it is very powerful for analyzing transient signals/images for its capability of multiresolution analysis with localization in both time and frequency domains. The wavelet-based multiresolution analysis is very efficient in pattern recognition, image compression, and image denoising, but there is no guarantee for all these denoising techniques that edges can be preserved well when high PSNR is achieved for the denoised image. Motivated by the above mentioned points, this paper develops an effective optimized wavelet-based denoising approach. 
Table 1. Review on Image Denoising by Wavelet Transform.

\begin{tabular}{|c|c|c|c|}
\hline $\begin{array}{l}\text { Author } \\
\text { [Citation] }\end{array}$ & $\begin{array}{l}\text { Adopted } \\
\text { Methodology }\end{array}$ & Features & Challenges \\
\hline $\begin{array}{l}\text { Xiang et al. } \\
(2017)\end{array}$ & $\begin{array}{l}\text { Stationary wavelet } \\
\text { transform }\end{array}$ & $\begin{array}{l}* \text { High accuracy } \\
* \text { High stability }\end{array}$ & $\begin{array}{l}\text { Presence of redundancy } \\
\text { Increased computational } \\
\text { complexity }\end{array}$ \\
\hline $\begin{array}{l}\text { Li et al. } \\
(2016)\end{array}$ & $\begin{array}{l}\text { Contourlet } \\
\text { transform }\end{array}$ & $\begin{array}{l}\text { * Better restoration quality } \\
\text { High PSNR value }\end{array}$ & $\begin{array}{l}\text { Requires more calculation time } \\
\text { Its basic images are localized in the } \\
\text { frequency domain }\end{array}$ \\
\hline $\begin{array}{l}\text { Remenyi et al. } \\
\text { (2014) }\end{array}$ & $\begin{array}{l}\text { Complex wavelet } \\
\text { transform }\end{array}$ & $\begin{array}{l}\text { Excellent quantitative and } \\
\text { visual performance } \\
\text { Conceptually simple and } \\
\text { practically efficient algorithm }\end{array}$ & $\begin{array}{l}\text { Failed to perform threshold limit } \\
\text { selection based on statistical models } \\
\text { of wavelet coefficients }\end{array}$ \\
\hline $\begin{array}{l}\text { Jesus et al. } \\
(2015)\end{array}$ & $\begin{array}{l}\text { Haar wavelet } \\
\text { transform }\end{array}$ & $\begin{array}{l}\text { Drastically reduces the noise } \\
\text { Improved variance } \\
\text { stabilization }\end{array}$ & $\begin{array}{l}\text { It is continuous and therefore non- } \\
\text { differentiable } \\
\text { It can't compress the energy of } \\
\text { the original signal into a few high- } \\
\text { energy values }\end{array}$ \\
\hline $\begin{array}{l}\text { Sun et al. } \\
(2014)\end{array}$ & $\begin{array}{l}\text { Bayesian MAP } \\
\text { estimation }\end{array}$ & $\begin{array}{l}\text { Effective method to remove } \\
\text { noise } \\
\text { * Possible to obtain sharp edges } \\
\text { and clear textures } \\
\text { * High quality }\end{array}$ & $\begin{array}{l}\text { * Information acquired is } \\
\text { theoretically infeasible } \\
\text { * Not automatic and computationally } \\
\text { infeasible }\end{array}$ \\
\hline $\begin{array}{l}\text { Song et al. } \\
(2016)\end{array}$ & $\begin{array}{l}\text { Gradient } \\
\text { histogram }\end{array}$ & $\begin{array}{l}\text { Can easily restore the image } \\
\text { textures } \\
\text { Improved image quality }\end{array}$ & $\begin{array}{l}* \text { Less robustness } \\
* \text { Low detection speed }\end{array}$ \\
\hline $\begin{array}{l}\text { Naimi et al. } \\
\text { (2015) }\end{array}$ & DT-CWT & $\begin{array}{l}\text { High efficiency in denoising } \\
\text { Less redundant and easy } \\
\text { computation }\end{array}$ & $\begin{array}{l}\text { Long process leads take a long } \\
\text { time }\end{array}$ \\
\hline $\begin{array}{l}\text { Zhang et al. } \\
\text { (2013) }\end{array}$ & 2D-DWT & $\begin{array}{l}\text { Can better retain the required } \\
\text { details from the image } \\
* \text { Suppress the image noises }\end{array}$ & $\begin{array}{l}\text { - Greater complexity } \\
* \text { Difficulties in data interpretation }\end{array}$ \\
\hline
\end{tabular}

\section{BACKGROUND}

\section{Feature Extraction}

Image denoising procedure is carried out for five sets of images like texture, nature, medical, satellite, and other images. In order to extract features from each image, this paper uses Noise power spectra (NPS) and bark frequency after adding noise to the images, which are described as follows.

NPS: In general, NPS (Hanson, 1998) is also termed as Weiner spectrum, which is modeled as Fourier Transform of noise images that are given in Eq. (1) as a mathematical form, where $(x, y)$ indicates the position and $v(x, y)$ specifies the noise image. The bracket \langle\rangle denotes mean over an infinite ensemble of noise images. Over the area $\Delta x \Delta y$, integration 
is provided that extends to infinity. If the image is in 2-dimension frequency domain, NPS of an image is naturally in 2-dimension format. If the noise is Gaussian distributed regarding a zero mean value and stationary, the NPS can be termed as a method that provides effective characterization of the noise. Therefore, the covariance is independent of position in the image.

$$
N\left(f_{x}, f_{y}\right)=\lim _{\Delta x, \Delta y \rightarrow \infty} \frac{1}{\Delta x \Delta y}\left\langle\left|\int v(x, y) \exp \left[-2 \pi\left(y f_{y}+x f_{x}\right)\right] d x d y\right|^{2}\right\rangle
$$

The average of the power of Fourier transform of noisy image samples obtained from a single noisy image or multiple images is the estimation pattern of NPS. Through this method, gradual alternation in the image intensity is eliminated, and samples are windowed before taking the Fourier transform. However, it is a complex task to work with frequency region of NPS and its analysis. Let $M$ be the averaged samples, and relative rms deviation in the power in each 2-dimesnional frequency bin is almost $[M]^{\frac{1}{2}}$. Even though the method is complex, it generates the outcome with outright normalization that admits various laboratories.

In the spatial domain, the convolution of two images is proportionate to multiplication in the frequency domain Fourier transforms. Thus, the NPS of a noisy image is improved by convolving a noisy image with $a$ by a factor of $|A|^{2}$, where $a$ denotes the convolution kernel and $A$ refers to the Fourier Transform of $a$. As the total power in the frequency domain is equal to the spatial domain, Eq. (2) determines the variance of convolved image.

$$
\sigma_{a}^{2}=\int|A|^{2} N\left(f_{x}, f_{y}\right) d f_{x} d f_{y}
$$

In the case of discretely sampled images, $-f_{M}$ to $f_{M}$ are the ranges of integration for both $f_{x}$ and $f_{y}$, where $f_{M}$ indicates the Nyquist frequency. The term $f_{M}$ is determined using Eq. (3), where $l$ denotes the pixel spacing in both $x$ and $y$ directions.

$$
f_{M}=[2 l]^{-1}
$$

The modified equation of Eq. (2) is provided in Eq. (4), where $K_{a}$ indicates the normalization constant and the bracket \langle\rangle refers to an average function of the power spectrum of $a$, which is weighted by $|A|^{2}$.

$$
\langle N\rangle_{|A|^{2}}=\sigma_{a}^{2} l^{2} K_{a}
$$

The reciprocal of total power of kernel defines $K_{a}$, which is given in Eq. (5), where the elements of the convolution kernel for discretely sampled images are denoted as $a_{i, j}$.

$$
K_{a}^{-1}=\int|A|^{2} d f_{x} d f_{y}=\sum_{i, j} a_{i, j}^{2}
$$

Therefore, NPS $F_{1}$ of the image at concerned spatial frequencies is estimated by properly choosing not only the convolution kernel but also the variance in the convolved noise image.

Bark frequency: It is defined as "a frequency scale on which equal distances correspond with perpetually equal distances" (Chung et al., 2017). In fact, the critical band can be termed as bark scale, a perpetual measure that is associated with image frequency to the perpetual resolution, which is non-linear. Here, one critical band is covered by one bark. Eq. (6) specifies the logical expression of the mapping function from $\hat{f}$ frequency to $B$ bark frequency.

$$
F_{2}=B[\hat{f}]=13 \arctan [0.76 \hat{f}]+3.5 \arctan \left([\hat{f} / 7.5]^{2}\right)
$$

\section{Classification}

Before denoising the image, it is essential to analyze the type of the image. Here, BR classifier is used to classify the type of the image. BR is selected since it has been proven to have better predictive capability to reveal relationship 
between data of different data distributions (Aggarwal et al., 2005). The extracted features $F_{1}$ and $F_{2}$ are given as the input to BR classifier, which results in the respective type of image. The BR classifier model is explained in the below section.

\section{Optimization Of Filter Coefficients}

In this paper, the filter coefficient parameter of DT-CWT is optimally selected using GA algorithm (Call, 2005). The algorithm consists of six major steps. (i) Crossover, (ii) Mutation, (iii) Genotype-Phenotype Mapping, (iv) Fitness, (v) Selection, and (vi) Termination.

Crossover: This is an operator, which allows the permutation of the genetic factual of two or more solutions. Naturally, most species comprise two parents. Moreover, in some exceptional cases, there are no different sexes and thus they have only one parent. This operator implements an application, which combines the genetic factual of the parents. A famed one for bit string demonstration is $c$ point crossover. It splits into two solutions at $c$ positions and consecutively gathers them into one. The formulation for crossover is defined in Eq. (7) and Eq. (8), where $i$ indicates $i^{\text {th }}$ gene, $C_{p}$ indicates the crossover point, and $C_{r}$ indicates the crossover rate and $L$ indicates the length of chromosome.

$$
\begin{aligned}
& x^{\text {child }}(i)=\left\{\begin{array}{ll}
x_{1}(i) \text { if } & i<C_{p} \\
x_{2}(i) & \text { else }
\end{array}\right\} \\
& C_{p}=\left[C_{r} \times L\right]
\end{aligned}
$$

Mutation: This is the second step, in which the mutation operator alters the solutions by distributing them. Mutation works on the basis of random changes. The strength of this disruption is known as the mutation rate. Three major desires are there in the mutation operator. The initial condition is reachability. Every point in the solution space must be accessible or reachable from the random point in the solution space. The second condition is unbiasedness. The operator should not persuade a point of the exploration to a specific direction. The third condition or principle of this operator is scalability. Each mutation operator must grant the degree of freedom that its strength is adjustable. The formulation for mutation is in eq. (9), where $j \in\left(R^{1 \times N_{R}}\right),\left(R^{1 \times N_{R}}\right)$ indicates the real number with dimension $N_{R}$.

$$
x(j)=\operatorname{rand}\left(x_{i}\right)
$$

Genotype-Phenotype Mapping: The new offspring population has been estimated after the evaluation of crossover and mutation operators. The mapping of the chromosome (genotype) depends on the representation, named as phenotype. This type of genotype-phenotype mapping must avoid the bias introduction.

Fitness: In this step, the phenotype of solution is formulated on fitness model. This function measures the solution's quality, which is generated by this algorithm. Further, it is a part of modeling process of the entire optimization model. The performance of GA in problem-solving is measured in correspondence with the count of required fitness function assessments till the optimal is found.

Selection: The selection is a process that chooses the parents of the new generation, which is termed as survival selection. This operator defines which operator must survive and which operator must die. This perception directly implements the 'Darwin's Principle of survival'. However, the developed survival operator can be employed for mating selection, which is the part of the crossover operators. Moreover, mating strategy is a vital part, where it decides which parents should join in the crossover progression.

Termination: This condition defines when the major evolutionary loop terminates. GA often runs in the predefined count of generations. The time and cost of the fitness model estimation often restrict the length of the optimization procedure. 
The pseudo code of the GA algorithm is given in Algorithm 1 and the flow chart is illustrated in Fig. 1.

\begin{tabular}{|l|}
\hline \multicolumn{1}{|c|}{ Algorithm 1: GA-based Filter Coefficient optimization } \\
\hline Initialize the random $C$ population \\
Formulate the fitness Fit $(p)$ of each $p$ chromosome in the population \\
Repeat \\
Choose the 'best' entities that are to be utilized by the genetic operators \\
Generation of new entities using crossover and mutation operators. \\
Fitness evaluation of new entities \\
'Worst' entities are replaced by 'best' entities \\
Until it reaches the 'best' solution
\end{tabular}

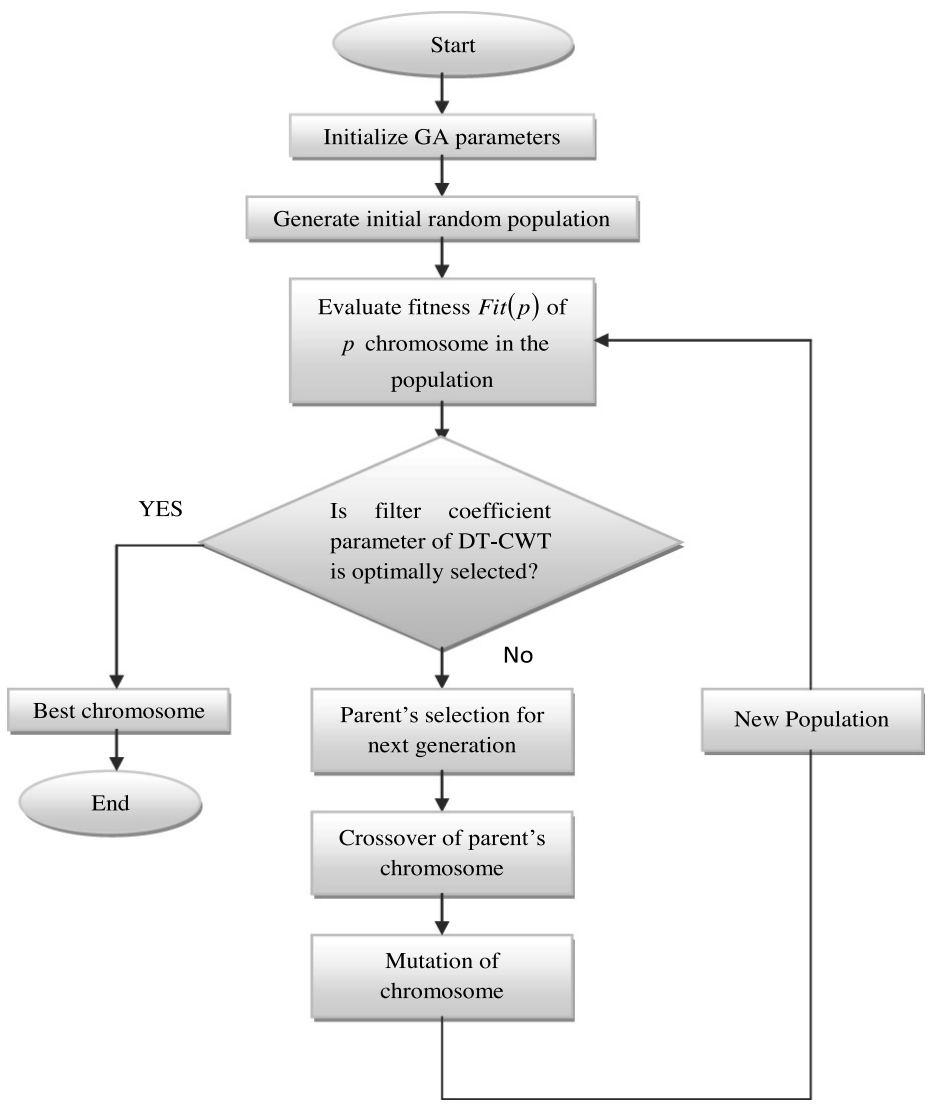

Fig. 1. Flow chart for GA- based filter coefficient optimization.

\section{Bayesian Regularization}

$\mathrm{BR}$ is a regularization technique that has the capability of obtaining the lower mean squared errors. The framework for neural network is on the basis of probabilistic interpretation of the parameters of the network and involves a probability distribution of network weights. In the BR network, the regularization adds subordinate term and objective function to penalize large weights for attaining better smoother mapping. The objective function as given in Eq. (11) is minimized using gradient-based optimization algorithm. 


$$
O=\beta M_{S}(I \mid W, N N)+\alpha S_{S}(W \mid N N)
$$

where $S_{S}(W \mid N N)$ is $S_{S}=\frac{1}{p} \sum_{k=1}^{p} W_{k}^{2}$, the sum of squares of network weights $W, \alpha$ and $\beta$ are the hyperparameters, $M_{S}$ denotes the mean sum of squares of the network error, $I=\left\{F_{1}, F_{2}\right\}$ specifies the input target features, $N N$ refers to the neural network architecture, which comprises the specification of count of layers, count of unit in every layer, and the activation function type, $\alpha S_{S}(W \backslash N N)$ is known as weight decay, and $\alpha$ is also termed as decay rate. If $\alpha<\beta$, then the algorithm minimizes the error, and the training of algorithm reduces the weight size if $\alpha \gg \beta$. The posterior $P_{D}$ distribution of weight is updated using Bayes' rule, which is defined in Eq. (12), where $P_{D}(W \mid \alpha, N N)$ denotes the prior distribution of weight, and $P_{D}(I \mid W, \beta, N N)$ denotes the likelihood function.

$$
P_{D}(W \mid I, \alpha, \beta, N N)=\frac{P_{D}(I \mid W, \beta, N N) \cdot P_{D}(W \mid \alpha, N N)}{P_{D}(I \mid \alpha, \beta, N N)}
$$

The optimized weight maximizes the posterior probability of $W$, which is equivalent to reducing the regularized objective function $O=\beta M_{S}+\alpha S_{S}$. The joint posterior density is defined as given in Eq. (13).

$$
P_{D}(\alpha, \beta \mid I, N N)=\frac{P_{D}(I \mid \alpha, \beta, N N) P_{D}(\alpha, \beta \mid N N)}{P_{D}(I \mid N N)}
$$

According to Mackay, Eq. (12) is determined as given in Eq. (13), where $p$ and $q$ specify the count of observation as well as the total count of parameters of network, respectively. Eq. (14) (Laplace approximation) grants Eq. (15), where $H^{M A P}$ denotes the Hessian matrix of the objective function and MAP stands for 'Maximum A Posteriori.'

$$
\begin{aligned}
& P_{D}(I \mid \alpha, \beta, N N)=\frac{P_{D}(I \mid W, \beta, N N) P_{D}(W \mid \alpha, N N)}{P_{D}(W \mid I, \alpha, \beta, N N)} \\
&=\frac{Z_{O}(\alpha, \beta)}{(\pi / \beta)^{p / 2}(\pi / \alpha)^{q / 2}} \\
& Z_{O}(\alpha, \beta)=\infty\left|H^{M A P}\right|^{-\frac{1}{2}} \exp \left(-O\left(W^{M A P}\right)\right)
\end{aligned}
$$

The parameter at $t$ iteration is updated as given in Eq. (16), where $\mu$ refers to the Levenberg damping factor, and $J$ denotes the jacobian matrix, which comprises the first derivative of the network errors in terms of network parameters. Further, $\mu$ is adjustable for all iterations, which leads to optimization.

$$
W^{t+1}=W^{t}-\left[J^{T} J+\mu I\right]^{-1} J^{T} e
$$

Therefore, BR classifier automatically predicts the filter coefficient that to be given to DT-CWT for denoising each image.

To analyze the performance of the proposed model, the performance metrics such as PSNR, SDME, SSIM, MSE, RMSE, MAE, PC, and SMAPE are used, where the mathematical formulation is given in Table 2 (Srivastava, 2014). 
Table 2. Performance metrics utilized for analysis.

\begin{tabular}{|c|c|}
\hline Performance Measures & Formulas \\
\hline PSNR & $20 \log _{10}\left[\frac{255}{R M S E}\right]$ \\
\hline SDME & $-\frac{1}{\hat{K}_{1} \hat{K}_{2}} 20 \ln \frac{\hat{I}_{\max : \hat{k}, \hat{l}}-2 \hat{I}_{\text {centrw: } k, \hat{l}}+\hat{I}_{\min : \hat{k}, \hat{l}}}{\hat{I}_{\max : \hat{k}, \hat{l}}+2 \hat{I}_{\text {centrw: } \hat{k}, \hat{l}}+\hat{I}_{\min : \hat{k}, \hat{l}}}$ \\
\hline SSIM & $\frac{\left(2 \mu_{\hat{X}} \mu_{\hat{Y}}+C_{1}\right)\left(2 \sigma_{\hat{X} \hat{Y}}+C_{2}\right)}{\left(\mu_{\hat{X}}^{2}+\mu_{\hat{Y}}^{2}+C_{1}\right)\left(\sigma_{\hat{X}}^{2}+{\sigma_{\hat{Y}}}^{2}+C_{1}\right)}$ \\
\hline MSE & $\frac{1}{\hat{N}} \sum_{\hat{i}, \hat{j}}\left(\hat{X}_{\hat{i}, \hat{j}}-\hat{Y}_{\hat{i}, \hat{j}}\right)^{2}$ \\
\hline RMSE & $\sqrt{\frac{1}{\hat{N}} \sum_{\hat{i}, \hat{j}}\left(\hat{X}_{\hat{i}, \hat{j}}-\hat{Y}_{\hat{i}, \hat{j}}\right)^{2}}$ \\
\hline MAE & $\frac{1}{\hat{N}} \sum_{\hat{i}, \hat{j}}\left|\hat{X}_{\hat{i}, \hat{j}}-\hat{Y}_{\hat{i}, \hat{j}}\right|$ \\
\hline \multirow[t]{2}{*}{$\mathrm{PC}$} & $\sum_{\hat{i}, \hat{j}=1}\left(\hat{X}_{\hat{i}}-\overline{\hat{X}}_{\hat{i}}\right)\left(\hat{Y}_{\hat{i}}-\overrightarrow{\hat{Y}}_{\hat{i}}\right)$ \\
\hline & $\sqrt{\sum_{i=1}^{N}\left(\hat{X}_{\hat{i}}-\overline{\hat{X}}_{\hat{i}}\right)^{2}} \sqrt{\sum_{j=1}^{N}\left(\hat{Y}_{\hat{i}}-\overline{\hat{Y}}_{\hat{i}}\right)^{2}}$ \\
\hline SMAPE & $\frac{1}{\hat{N}} \sum_{\hat{i}, \hat{j}} \frac{\left|\hat{X}_{\hat{i}, \hat{j}}-\hat{Y}_{\hat{i}, \hat{j}}\right|}{\hat{i}, \hat{j}|+| \hat{Y}_{\hat{i}, \hat{j}} \mid}$ \\
\hline
\end{tabular}

\section{PROPOSED METHODOLOGY}

\section{Proposed Architecture}

Fig. 2 illustrates the block representation of the proposed DT-CWT based image denoising model. This model intends to denoise the input image, and the procedure is as follows: the preliminary process is adding noise to the input image, by which the effective denoising of image can be achieved. This work mainly contributes two tasks: (i) classification of image type using BR and (ii) denoising the image by estimating the optimal wavelet coefficient to DT-CWT using GA. In the first contribution, the extracted $F_{1}$ and $F_{2}$ features are given as the input to BR classify the corresponding image. 


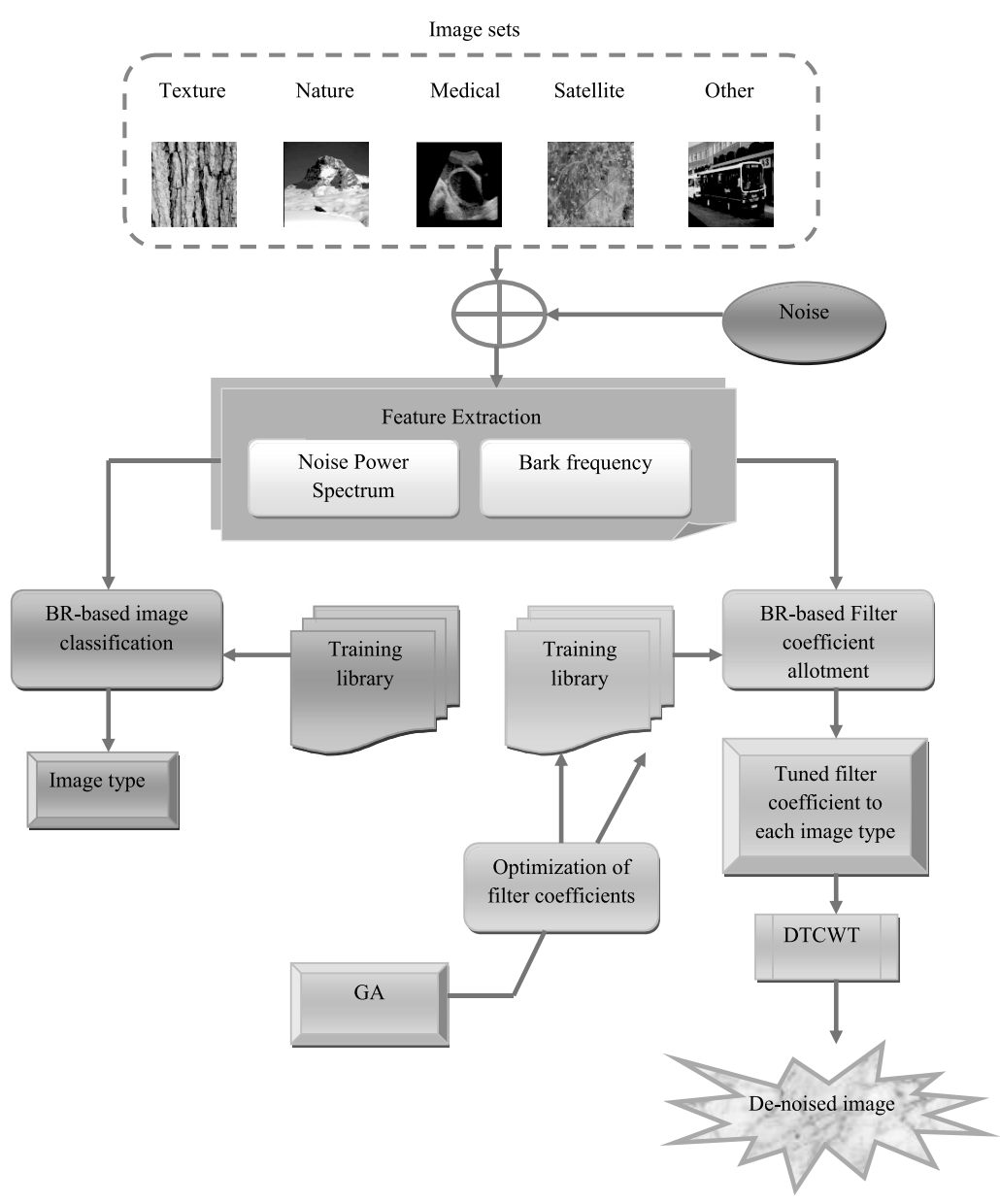

Fig. 2. Block diagram of proposed image denoising model.

On the other hand, the contribution is made in solving the problem of determining the optimal 'filter coefficient' to increase the PSNR value. Here, GA algorithm is used to identify the apt filter coefficient. The objective function of the proposed model is defined in Eq. (17).

$$
D=\operatorname{Max}\{P S N R\}
$$

\section{DT-CWT Model}

DT-CWT (YueSi et al., 2016; Selesnick et al., 2005; Mohan et al., 2016; Sunil et al., 2016) is one of the effective models for the application of analytic wavelet transform. This model consists of two real DWTs: the first DWT grants the real part of transform, whereas the second grants the imaginary part. Further, the model grants perfect reconstruction and directional discernment and is independent of count of scales. The diagrammatic representation of DT-CWT is illustrated in Fig 3, where $L_{0}(n)$ and $L_{1}(n)$ refer to the low pass/high pass filter pair for upper Filter Bank (FB) and $H_{0}(n)$ and $H_{1}(n)$ denotes the low pass/ high pass filter pair for lower FB, respectively. 


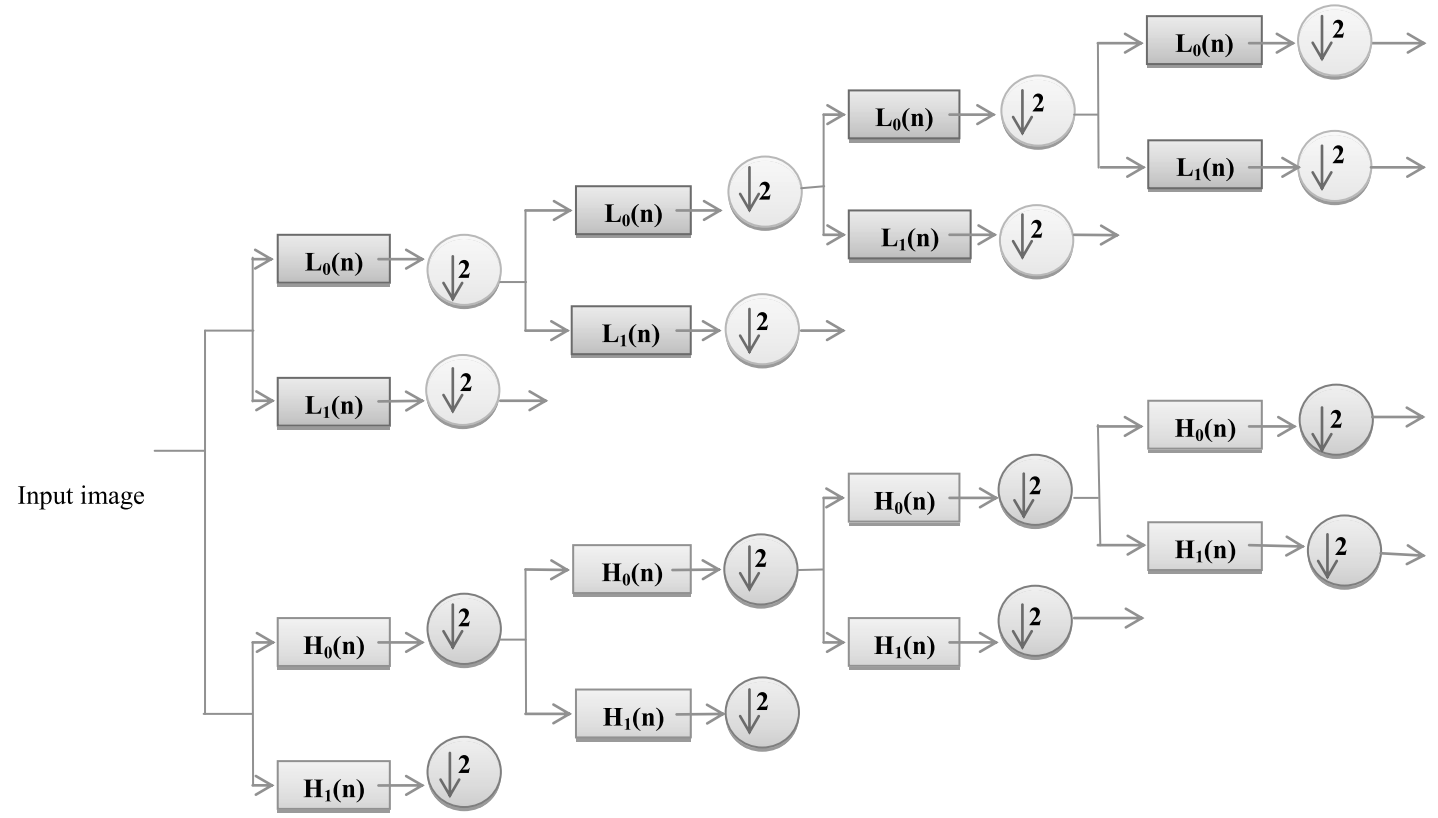

Fig. 3. Diagrammatic representation of DT-CWT model.

Consistent with wavelet theory, both wavelet coefficients $w_{i}^{\text {real }}(K)$ and scaling coefficients $s_{j}^{\text {real }}(K)$ of the real part transform are attained through inner products, which are defined in Eq. (18) and Eq. (19), where $i$ refers to the scale factor, $j$ denotes the decomposition level, and $\omega_{h}$ and $\phi_{h}$ denote the wavelet functions of real part transform. Further, the coefficients of the imaginary part transform can be formulated as defined in Eq. (20) and (21).

$$
\begin{aligned}
& w_{i}^{\text {real }}(K)=2^{i / 2} \int_{-\infty}^{+\infty} Y(u) \omega_{h}\left(2^{i} u-K\right) d u \quad i=1, \ldots j \\
& s_{j}^{\text {real }}(K)=2^{j / 2} \int_{-\infty}^{+\infty} Y(u) \phi_{h}\left(2^{j} u-K\right) d u \\
& w_{i}^{\text {imaginary }}(K)=2^{i / 2} \int_{-\infty}^{+\infty} Y(u) \omega_{h}\left(2^{i} u-K\right) d u i=1, \ldots . j \\
& s_{j}^{\text {imaginary }}(K)=2^{j / 2} \int_{-\infty}^{+\infty} Y(u) \phi_{g}\left(2^{j} u-K\right) d u
\end{aligned}
$$

Hence, the wavelet as well as the scaling coefficients of DT-CWT are acquired as defined in Eq. (22) and (23), respectively. Then the wavelet or scaling coefficients are reconstructed as defined in Eq. (24) and (25), respectively.

$$
\begin{aligned}
& w_{i}^{s}(K)=w_{i}^{\text {real }}(K)+j w_{i}^{\text {imaginary }}(K) i=1, \ldots \ldots . j \\
& s_{j}^{s}(K)=s_{i}^{\text {real }}(K)+j s_{j}^{\text {imaginary }}(K) \\
& w_{i}(u)=2^{(i-1) / 2}\left[\sum_{n} w_{i}^{\text {real }}(K) \omega_{h}\left(2^{i} u-n\right)+\sum_{m} w_{i}^{\text {imaginary }}(K) \omega_{g}\left(2^{i} u-m\right)\right] \\
& i=1, . . j \\
& s_{j}(u)=2^{(j-1) / 2}\left[\sum_{n} s_{j}^{\text {real }}(K) \phi_{h}\left(2^{j} u-n\right)+\sum_{m} s_{j}^{\text {imaginary }}(K) \phi_{g}\left(2^{j} u-m\right)\right]
\end{aligned}
$$


DT-CWT decomposes the vibration signal $Y(u)$, which is defined as given in Eq. (26), where $w_{i}(u) i=1,2 \ldots . j$ and $s_{j}(u)$ denotes the sub-band signals that are organized from high to low frequency.

$$
Y(u)=s_{j}(u)+\sum_{i=1}^{j} w_{i}(u)
$$

The application of 2D DT-CWT comprises two major steps: Initially, the input image is decomposed up to certain levels via two 2D-DWT branches, $a$ and $b$. At each level, six high pass sub-bands are engendered like $H L_{a}, L H_{a}, H H_{a}, H L_{b}, L H_{b}$ and $H H_{b}$. Next to this, the respective sub-bands that have same pass bands are combined linearly through averaging or differencing. As a result of this, the sub-bands of 2D DT-CWT at all levels are generated as defined in Eq. (27)

The six wavelets defined here have sum or difference operation, which is orthonormal. Further, the 2D DT-DWT has same basis function and real part, and the structure has the extension of conjugate filtering.

$$
\begin{aligned}
& \left(L H_{a}+L H_{b}\right) / \sqrt{2},\left(L H_{a}-L H_{b}\right) / \sqrt{2},\left(H L_{a}+H L_{b}\right) / \sqrt{2} \\
& \left(H L_{a}-H L_{b}\right) / \sqrt{2},\left(H H_{a}+H H_{b}\right) / \sqrt{2},\left(H H_{a}-H H_{b}\right) / \sqrt{2}
\end{aligned}
$$

Among the analysis filter, the low pass $H_{L}(n)$ and high $H_{H}(n)$ pass filters of first level coefficients and low pass $H_{S}(n)$ of second level coefficients are given as solutions for optimization since the mentioned coefficients give more informative details about the image. The solution encoding is diagrammatically illustrated in Fig. 4, where $n=1, \ldots N_{S}$, $N_{S}$ denotes the number of samples.

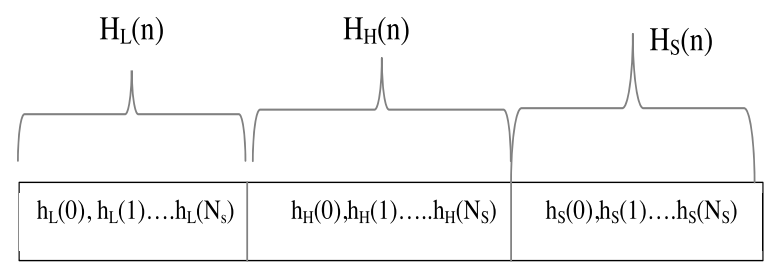

Fig. 4. Representation of solution encoding.

\section{RESULTS AND DISCUSSIONS}

\section{Simulation Setup}

The developed intelligent image classification was carried out in MATLAB 2015a. Five image sets were chosen, namely, Texture image set, Nature image set, Medical image set, Satellite image set, and Miscellaneous image set. The texture images were downloaded from (http://www-cvr.ai.uiuc.edu/ponce_grp/data/: access data 2019-0215), medical images were downloaded from (http://www.ultrasoundcases.info/case-list.aspx?cat=26: access date 2019-02-15), satellite images were manually collected, natural images were downloaded from (http://www-cvr. ai.uiuc.edu/ponce_grp/data/: access data 2019-02-15), and miscellaneous images were downloaded from two links (http://vismod.media.mit.edu/pub/VisTex/, https://sites.google.com/site/dctresearch/Home/content-based-imageretrieval: access data 2019-02-15). The images Features like NPS and Bark frequency were extracted from the image. The classification was done using BR classifier. Moreover, the classifier was trained using the renowned GA algorithm, where the optimized filter coefficients of DT-CWT were generated. The performance of the proposed model was analyzed in terms of measures like PSNR, SDME, SSIM, MSE, RMSE, MAE, PC, and SMAPE, respectively. Further, the developed model was compared to some of the conventional models like 2D SMCWT, DT-CWT, DT-CDWT, DT-RDWT, W-ST, and W-HT in terms of efficient image denoising. Fig 5 illustrates the experimental results for five image sets using proposed and conventional models. 


\begin{tabular}{|c|c|c|c|c|c|}
\hline \multirow[b]{2}{*}{ Models } & \multicolumn{5}{|c|}{ Image sets } \\
\hline & Texture Image & Nature image & Medical image & Satellite image & $\begin{array}{l}\text { Miscellaneous } \\
\text { image }\end{array}$ \\
\hline (a) & & & & & \\
\hline (b) & & & & & \\
\hline (c) & & & & & \\
\hline & & E: & & & III \\
\hline & 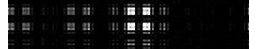 & 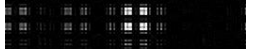 & in: & : & ia: \\
\hline (d) & & & & & \\
\hline (e) & 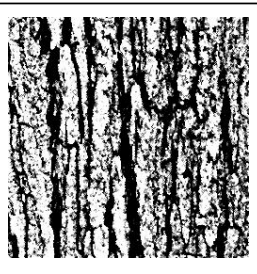 & 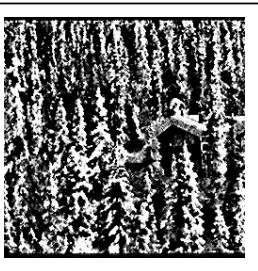 & 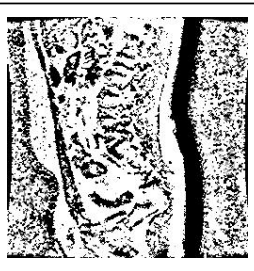 & 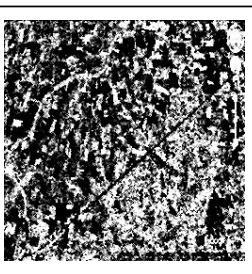 & \\
\hline (f) & 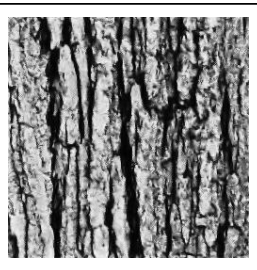 & 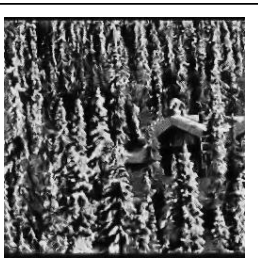 & $\sqrt{a}+3$ & 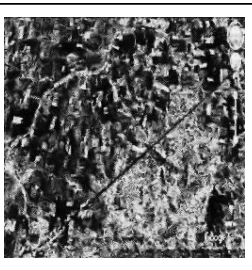 & 18] \\
\hline (g) & 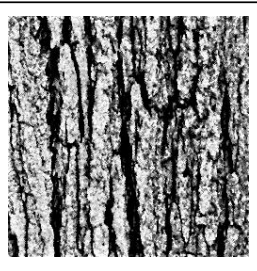 & 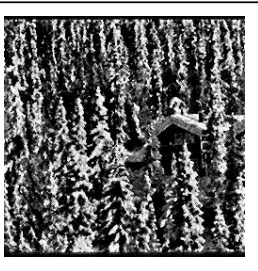 & 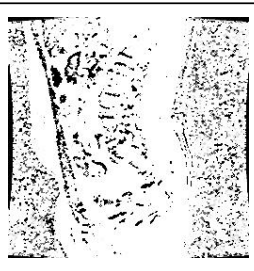 & 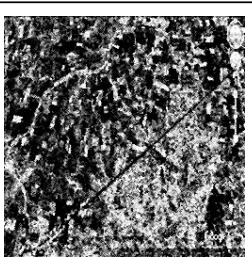 & $\frac{1}{1 \cdot 1}$ \\
\hline
\end{tabular}




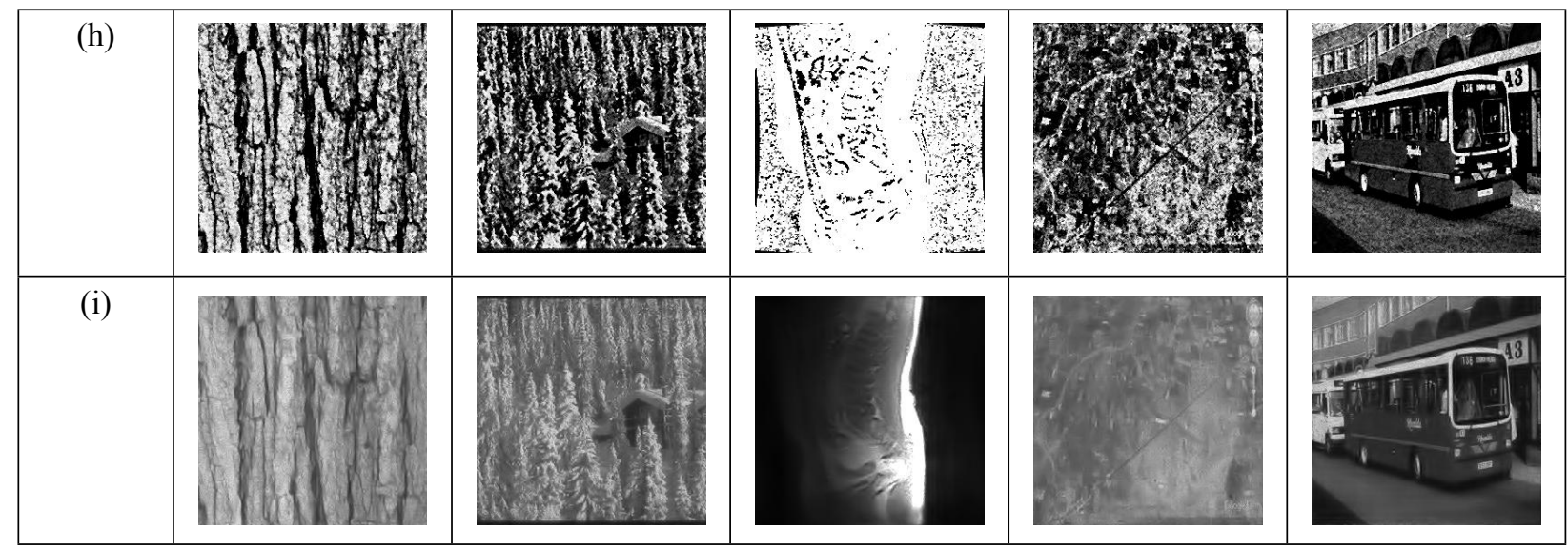

Fig. 5. Experimental results using five image sets for proposed Denoising model over the conventional models for a) Original image, b) Noisy image, c) 2D SMCWT-based denoising, d) DT-CWT-based denoising, e) DT-CDWT-based denoising, f) DT-RDWT-based denoising, g) W-ST-based denoising,

h) W-HT-based denoising, and i) Proposed image denoising.

\section{Qualitative Assesment}

Table 3 shows the performance analysis of proposed model over conventional methods in terms of various analytical measures. The analysis has been made for all the five image sets. From Table 3, it is observed that, for texture image, the proposed model has attained promising results. PSNR of the developed model is $69.97 \%, 5.85 \%$, $76.91 \%, 33.38 \%, 46.40 \%$, and $46.44 \%$ better than 2D SMCWT, DT-CWT, DT-CDWT, DT-RDWT,W-ST, and W-HT, respectively. The SDME of the proposed model is $97.42 \%, 41.26 \%, 98.64 \%, 89.66 \%, 93.88 \%$, and $93.89 \%$ superior to 2D SMCWT, DT-CWT, DT-CDWT, DT-RDWT, W-ST, and W-HT, respectively. Similarly, for SSIM measure, the proposed model has great deviation over conventional methods, and the model is $19.17 \%, 83.66 \%$, 24.65\%, 72.99\%, and 73.15\% better than DT-CWT, DT-CDWT, DT-RDWT, W-ST, and W-HT, respectively. The MSE of the proposed model is $97.05 \%, 27.31 \%, 97.83 \%, 81.30 \%, 90.11 \%$, and $90.12 \%$ better than 2D SMCWT, DT-CWT, DT-CDWT, DT-RDWT, W-ST, and W-HT, respectively. The MAE of the model is $12.33 \%, 86.39 \%$, $57.68 \%, 70.13 \%$, and $70.16 \%$ better than DT-CWT, DT-CDWT, DT-RDWT, W-ST, and W-HT, respectively. The PC of the proposed model is $98.24 \%, 11.73 \%, 22.51 \%, 10.99 \%, 17.51 \%$, and $17.53 \%$ superior to 2D SMCWT, DTCWT, DT-CDWT, DT-RDWT, W-ST, and W-HT, respectively. For SMAPE measure, the proposed model is $90.50 \%$, $10.11 \%, 84.61 \%, 65.46 \%, 79.40 \%$, and $79.43 \%$ superior to 2D SMCWT, DT-CWT, DT-CDWT, DT-RDWT, W-ST, and W-HT, respectively. For nature image set, it is observed that the PSNR of the proposed model is $67.16 \%$ and $12.71 \%$ better than 2D SMCWT and DTCWT, respectively. The same analysis is made for all the other image sets and it is clearly tabulated in Table 3. Altogether, it is reviewed that the performance of the proposed model is more efficient than that of the conventional methods by denoising the images more precisely. 
Table 3. Performance Of Proposed Model Versus Conventional Methods In Terms Of Various Analytical Measures.

\begin{tabular}{|c|c|c|c|c|c|c|c|c|}
\hline \multicolumn{9}{|c|}{ Texture images } \\
\hline Methods & PSNR & SDME & SSIM & MSE & RMSE & MAE & $\mathrm{PC}$ & SMAPE \\
\hline W-HT & 11.679 & -0.285 & 0.3172 & 4551.4 & 67.093 & 57.35 & 47326 & 0.858 \\
\hline W-ST & 11.686 & -0.285 & 0.317 & 4543.9 & 67.037 & 57.31 & 47336 & 0.857 \\
\hline DT-RDWT & 14.526 & -0.168 & 0.4406 & 2403.2 & 48.549 & 40.45 & 51081 & 0.511 \\
\hline DT-CDWT & 5.0333 & -1.288 & 0.089 & 20784.9 & 143.79 & 125.80 & 44469 & 1.148 \\
\hline DT-CWT & 20.530 & -0.029 & 0.460 & 618.2 & 24.466 & 19.52 & 50655 & 0.196 \\
\hline 2D-SMCWT & 6.5477 & -0.675 & -0.000 & 15246.1 & 122.01 & 114.6 & 1009.4 & 1.860 \\
\hline Proposed & 21.806 & -0.017 & 0.549 & 449.37 & 20.996 & 17.11 & 57391 & 0.176 \\
\hline \multicolumn{9}{|c|}{ Natural images } \\
\hline W-HT & -1.804 & -0.155 & 0.072 & 6524 & 2940 & 1850.3 & 21722 & 0.1528 \\
\hline W-ST & -1.801 & -0.155 & 0.072 & 65249 & 2940 & 1850.3 & 21726 & 0.1525 \\
\hline DT-RDWT & -0.950 & -0.124 & 0.098 & 65193 & 2933.8 & 1845.5 & 23300 & 0.0946 \\
\hline DT-CDWT & -4.067 & -0.359 & 0.0210 & $1.34 \times 10^{8}$ & 4231.3 & 2655.1 & 18633 & 0.2069 \\
\hline DT-CWT & 8.4888 & -0.069 & 0.1502 & 402.69 & 12.170 & 8.8928 & 2405 & 0.0344 \\
\hline 2D-SMCWT & 3.1950 & -0.284 & -0.0005 & 6177.4 & 49.188 & 40.98 & 51.835 & 0.3533 \\
\hline Proposed & 9.731 & -0.042 & 0.161 & 171.89 & 8.333 & 6.2906 & 26271 & 0.0320 \\
\hline \multicolumn{9}{|c|}{ Medical images } \\
\hline W-HT & 6.55642 & -0.11516 & 0.127394 & 940.8481 & 19.47514 & 14.13926 & 22840.94 & 0.068702 \\
\hline W-ST & 6.560875 & -0.11507 & 0.127369 & 938.7568 & 19.45269 & 14.12093 & 22848.89 & 0.068547 \\
\hline DT-RDWT & 8.140015 & -0.08393 & 0.149509 & 424.1882 & 12.89597 & 9.224428 & 25148.3 & 0.041373 \\
\hline DT-CDWT & 3.602328 & -0.25176 & 0.027016 & 4367.145 & 42.48501 & 32.72749 & 19773.04 & 0.103215 \\
\hline DT-CWT & 8.916861 & -0.07111 & 0.136066 & 255.6865 & 10.22897 & 7.852905 & 25485.98 & 0.023244 \\
\hline 2D-SMCWT & 3.493316 & -0.28554 & -0.00025 & 5573.821 & 45.89287 & 38.69709 & 352.3181 & 0.205667 \\
\hline Proposed & 10.07988 & -0.04745 & 0.151369 & 137.3159 & 7.489622 & 5.85446 & 27093.47 & 0.019075 \\
\hline \multicolumn{9}{|c|}{ Satellite images } \\
\hline W-HT & 1.662 & -0.037 & 0.025 & 659.64 & 9.645 & 8.165 & 5820.7 & 0.119 \\
\hline W-ST & 1.663 & -0.037 & 0.025 & 658.44 & 9.636 & 8.157 & 5822.6 & 0.119 \\
\hline DT-RDWT & 2.120 & -0.024 & 0.039 & 319.16 & 6.6905 & 5.513 & 6482.01 & 0.067 \\
\hline DT-CDWT & 0.751 & -0.135 & 0.007 & 2847.8 & 20.066 & 17.112 & 5356.5 & 0.165 \\
\hline DT-CWT & 3.548 & -0.006 & 0.055 & 34.10 & 2.152 & 1.644 & 7237.9 & 0.018 \\
\hline 2D-SMCWT & 1.304 & -0.094 & -0.0001 & 1249 & 13.085 & 12.52 & 48.319 & 0.258 \\
\hline Proposed & 3.561 & -0.004 & 0.052 & 31.76 & 2.101 & 1.666 & 7953.7 & 0.019 \\
\hline \multicolumn{9}{|c|}{ Miscellaneous images } \\
\hline W-HT & 4.916 & -0.41 & 0.216 & 9316 & 3678.7 & 2017.3 & 47149.2 & 0.3490 \\
\hline W-ST & 4.923 & -0.409 & 0.216 & 9316 & 3678.7 & 2017.3 & 47161.7 & 0.3474 \\
\hline DT-RDWT & 7.420 & -0.341 & 0.317 & 9310 & 3666.8 & 2008.1 & 50708.8 & 0.1606 \\
\hline DT-CDWT & -0.44 & -0.831 & 0.036 & $1.78 \mathrm{E}+08$ & 5112.5 & 2912.8 & 40409.4 & 0.5231 \\
\hline DT-CWT & 15.267 & -0.191 & 0.285 & 4879.1 & 42.60 & 31.891 & 50612.3 & 0.1544 \\
\hline 2D-SMCWT & 5.323 & -0.543 & -0.001 & 57069.3 & 152.35 & 107.94 & 434.49 & 0.8337 \\
\hline Proposed & 17.987 & -0.168 & 0.3532 & 2461.4 & 29.72 & 22.35 & 54235.7 & 0.1289 \\
\hline
\end{tabular}




\section{Adaptiveness in DT-CWT}

Table 4 shows the comparison of proposed model over the conventional DT-CWT method by varying noise levels as $0.02,0.04,0.05,0.06$, and 0.08 , respectively. The analysis is made in terms of various measures like PSNR, SDME, SSIM, MSE, RMSE, MAE, PC, and SMAPE, respectively; its formula is provided in table 2. For texture image set, it is observed that the proposed model has attained better PSNR at noise variance 0.02 , which is $69.19 \%$ superior to DT-CWT, respectively. At 0.04 noise variance, the proposed model is $68.38 \%$ better than DT-CWT, respectively. Similarly, for $0.05,0.06$, and 0.08 noise variance, the proposed model is $68.03 \%, 67.71 \%$, and $67.06 \%$ superior to DT-CWT, respectively. For nature image set, the PSNR of proposed model for noise variance $0.02,0.04,0.05,0.06$, and 0.08 is $63.88 \%, 62.70 \%, 62.33 \%, 61.84 \%$, and $61.00 \%$ superior to DT-CWT, respectively. For medical image set, the PSNR of proposed model for noise variance $0.02,0.04,0.05,0.06$, and 0.08 is $62.71 \%, 61.35 \%, 60.74 \%$, $60.20 \%$, and $59.13 \%$ better than conventional DT-CWT, respectively. For satellite image set, the PSNR of proposed model for noise variance $0.02,0.04,0.05,0.06$, and 0.08 is $64.67 \%, 63.68 \%, 63.27 \%, 62.85 \%$, and $62.20 \%$ better than conventional DT-CWT, respectively. For miscellaneous image sets, the PSNR of proposed model for noise variance $0.02,0.04,0.05,0.06$, and 0.08 is $66.81 \%, 65.61 \%, 65.04 \%, 64.50 \%$, and $63.53 \%$ better than conventional DT-CWT, respectively. The investigation is made for all the remaining measures by varying noise levels, and from the results it is observed that the proposed image denoising model is more efficient than the conventional DT-CWT.

Table 4. Performance Of Proposed Versus Conventional DT-CWT In Image Denoising By Varying Noise.

\begin{tabular}{|c|c|c|c|c|c|c|c|c|c|c|c|c|c|c|c|c|}
\hline \multicolumn{17}{|c|}{ Texture images } \\
\hline \multirow{2}{*}{$\begin{array}{c}\text { Noise } \\
\text { variance }\end{array}$} & \multicolumn{2}{|c|}{ PSNR } & \multicolumn{2}{|c|}{ SDME } & \multicolumn{2}{|c|}{ SSIM } & \multicolumn{2}{|c|}{ MSE } & \multicolumn{2}{|c|}{ RMSE } & \multicolumn{2}{|c|}{ MAE } & \multicolumn{2}{|c|}{$\mathrm{PC}$} & \multicolumn{2}{|c|}{ SMAPE } \\
\hline & Proposed & DT-CWT & Proposed & \begin{tabular}{|l|} 
DT-CWT \\
\end{tabular} & Proposed & $\begin{array}{c}\text { DT-CWT } \\
\times 10^{-3} \\
\end{array}$ & Proposed & DT-CWT & Proposed & DT-CWT & Proposed & DT-CWT & Proposed & DT-CWT & Proposed & \begin{tabular}{|l} 
DT-CWT \\
\end{tabular} \\
\hline 0.02 & 21.26 & 6.55 & -0.02 & -0.68 & 0.53 & -0.25 & 527.12 & 15241.62 & 22.53885 & 122.0009 & 18.09 & 114.64 & 54753.43 & 1016.23 & 0.18 & 1.87 \\
\hline 0.04 & 20.72 & 6.55 & -0.03 & -0.68 & 0.48 & -0.24 & 590.43 & 15244.79 & 23.92084 & 122.014 & 19.10 & 114.64 & 51752.87 & 1009.98 & 0.19 & 1.86 \\
\hline 0.05 & 20.49 & 6.55 & -0.03 & -0.68 & 0.46 & -0.24 & 620.24 & 15246.14 & 24.54043 & 122.0194 & 19.57 & 114.64 & 50448.08 & 1006.39 & 0.20 & 1.86 \\
\hline 0.06 & 20.29 & 6.55 & -0.03 & -0.67 & 0.44 & -0.24 & 647.68 & 15247.36 & 25.10048 & 122.0245 & 19.99 & 114.64 & 49280.9 & 1006.52 & 0.20 & 1.86 \\
\hline 0.08 & 19.89 & 6.55 & -0.04 & -0.67 & 0.41 & -0.25 & 705.62 & 15250.81 & 26.23163 & 122.0387 & 20.88 & 114.64 & 47042.65 & 1007.97 & 0.21 & 1.85 \\
\hline \multicolumn{17}{|c|}{ Nature images } \\
\hline 0.02 & 8.86 & 3.20 & -0.06 & -0.28 & 0.18 & -0.57 & 338.92 & 6176.43 & \begin{tabular}{|l|}
11.09566 \\
\end{tabular} & 49.18246 & 8.24 & 40.98 & 25017.99 & 53.56 & 0.03 & 0.37 \\
\hline 0.04 & 8.58 & 3.20 & -0.07 & -0.29 & 0.16 & -0.53 & 382.43 & 6176.77 & 11.86273 & 49.18499 & 8.70 & 40.98 & 24343.78 & 54.82 & 0.04 & 0.37 \\
\hline 0.05 & 8.47 & 3.19 & -0.07 & -0.28 & 0.15 & -0.55 & 403.76 & 6177.66 & 12.21283 & 49.18861 & 8.91 & 40.98 & 24023.21 & 49.11 & 0.03 & 0.34 \\
\hline 0.06 & 8.36 & 3.19 & -0.07 & -0.28 & 0.14 & -0.58 & 424.28 & 6177.90 & 12.54011 & 49.19018 & 9.12 & 40.99 & 23728.36 & 48.53 & 0.03 & 0.34 \\
\hline 0.08 & 8.18 & 3.19 & -0.07 & -0.29 & 0.13 & -0.54 & 464.05 & 6178.70 & 13.14225 & 49.19393 & 9.49 & 40.99 & 23172.14 & 53.16 & 0.04 & 0.34 \\
\hline \multicolumn{17}{|c|}{ Medical images } \\
\hline 0.02 & 9.36 & 3.49 & -0.07 & -0.29 & 0.16 & -0.23 & 200.91 & 5572.76 & 9.069204 & 45.88684 & 7.08 & 38.68 & 26147.92 & 350.59 & 0.02 & 0.20 \\
\hline 0.04 & 9.03 & 3.49 & -0.07 & -0.28 & 0.14 & -0.25 & 238.76 & 5573.29 & 9.908618 & 45.89021 & 7.64 & 38.69 & 25698.37 & 351.51 & 0.02 & 0.21 \\
\hline 0.05 & 8.89 & 3.49 & -0.07 & -0.29 & 0.13 & -0.24 & 256.55 & 5573.72 & 10.27418 & 45.89236 & 7.89 & 38.70 & 25480.5 & 349.43 & 0.02 & 0.21 \\
\hline 0.06 & 8.77 & 3.49 & -0.07 & -0.29 & 0.13 & -0.26 & 273.31 & 5574.07 & 10.61344 & 45.89452 & 8.11 & 38.70 & 25268.58 & 353.75 & 0.02 & 0.21 \\
\hline 0.08 & 8.54 & 3.49 & -0.06 & -0.28 & 0.12 & -0.26 & 308.90 & 5575.26 & 11.2794 & 45.90044 & 8.55 & 38.71 & 24834.53 & 356.31 & 0.02 & 0.20 \\
\hline \multicolumn{17}{|c|}{ Satellite images } \\
\hline 0.02 & 3.68 & 1.30 & -0.004 & -0.09 & 0.07 & -0.15 & 27.83 & 1248.75 & 1.943528 & 13.08382 & 1.50 & 12.53 & 7857.40 & 48.92 & 0.02 & 0.25 \\
\hline 0.04 & 3.58 & 1.30 & -0.006 & -0.09 & 0.06 & -0.14 & 32.22 & 1248.91 & 2.095634 & 13.08481 & 1.60 & 12.53 & 7395.54 & 47.94 & 0.02 & 0.27 \\
\hline 0.05 & 3.54 & 1.30 & -0.007 & -0.10 & 0.05 & -0.15 & 34.24 & 1249.07 & 2.161179 & 13.08556 & 1.65 & 12.53 & 7206.77 & 48.96 & 0.02 & 0.24 \\
\hline 0.06 & 3.50 & 1.30 & -0.006 & -0.09 & 0.05 & -0.14 & 36.28 & 1249.20 & 2.225776 & 13.08616 & 1.70 & 12.53 & 7019.21 & 47.11 & 0.02 & 0.27 \\
\hline 0.08 & 3.44 & 1.30 & -0.005 & -0.09 & 0.05 & -0.14 & 39.96 & 1249.42 & 2.335946 & 13.08741 & 1.78 & 12.53 & 6710.90 & 48.67 & 0.02 & 0.27 \\
\hline \multicolumn{17}{|c|}{ Miscellaneous images } \\
\hline 0.02 & 16.06 & 5.33 & -0.18 & -0.55 & 0.33 & -1.23 & 3876.75 & 57047.2 & 38.18263 & 152.3231 & 29.33 & 107.83 & 51957.58 & 435.78 & 0.13 & 0.81 \\
\hline 0.04 & 15.47 & 5.32 & -0.19 & -0.54 & 0.29 & -1.23 & 4548.27 & 57063.06 & 41.29973 & 152.3418 & 31.08 & 107.91 & 51027.08 & 430.90 & 0.16 & 0.86 \\
\hline 0.05 & 15.22 & 5.32 & -0.19 & -0.55 & 0.28 & -1.22 & 4876.63 & 57067.23 & 42.71521 & 152.3477 & 31.94 & 107.95 & 50589.7 & 441.38 & 0.17 & 0.85 \\
\hline 0.06 & 14.99 & 5.32 & -0.196 & -0.54 & 0.27 & -1.21 & 5221.81 & 57078.69 & 44.12461 & 152.3612 & 32.78 & 107.98 & 50152.76 & 428.84 & 0.16 & 0.80 \\
\hline 0.08 & 14.59 & 5.32 & -0.20 & -0.54 & 0.25 & -1.22 & 5872.17 & 57090.44 & 46.69325 & 152.3771 & 34.32 & 108.07 & \begin{tabular}{|l|}
49334.8 \\
\end{tabular} & 435.55 & 0.16 & 0.85 \\
\hline
\end{tabular}


Fig 6 shows the performance of the proposed model for texture image set over the conventional methods by varying noise levels from 0.02 to 0.08 . From Fig 6, it is observed that the PSNR of the proposed model for noise level 0.02 is $68.96 \%, 10.77 \%, 74.56 \%, 31.89 \%$, and $43.53 \%$ superior to 2D-SMCWT, DT-CWT, DT-CDWT, DT-RDWT, and W-ST, respectively. Similarly, SDME of the proposed model is $44.44 \%, 82.75 \%, 28.57 \%$, and $16.66 \%$ better than 2D-SMCWT, DT-CDWT, DT-RDWT, and W-ST, respectively. The analysis is also made for all the remaining measures. From the analysis, it is observed that the proposed model has attained high SSIM and PC when compared to the conventional methods. Further, the model has attained low MSE, RMSE, MAE, and SMAPE, which shows the betterment of the proposed model.

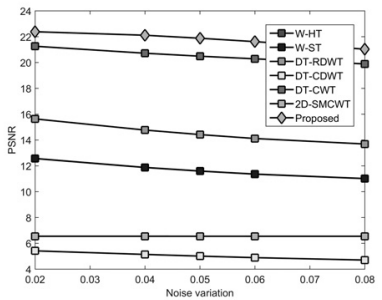

(a)

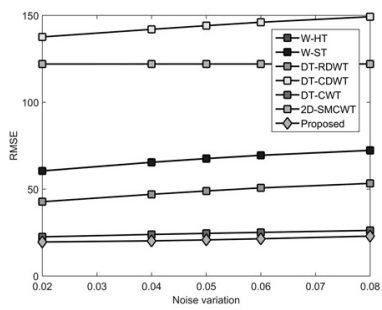

(e)

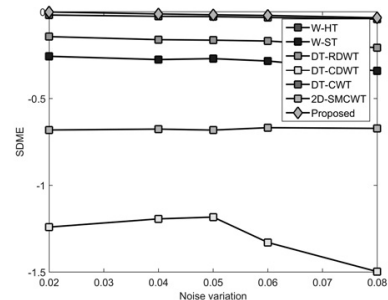

(b)

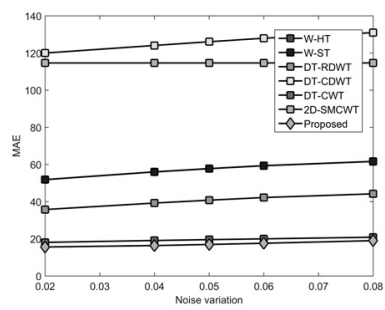

(f)

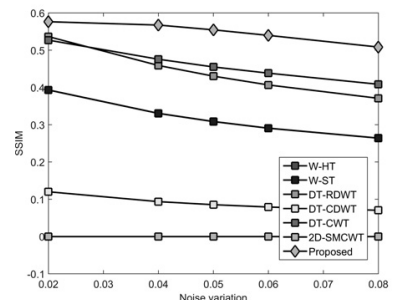

(c)

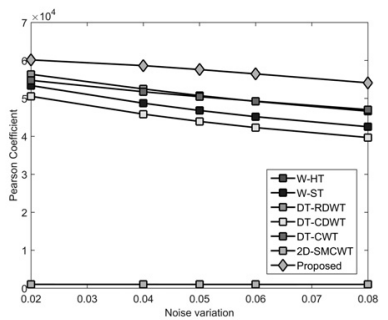

(g)

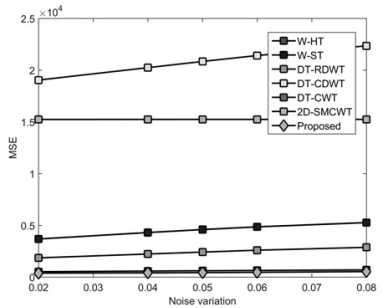

(d)

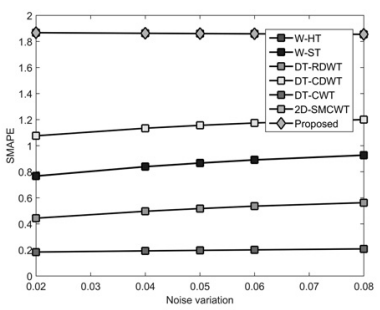

(h)

Fig. 6. Performance analysis of proposed image denoising model for Texture image set by varying noise in terms of (a) PSNR, (b) SDME, (c)SSIM, (d) MSE, (e) RMSE, (f) MAE, (g) PC, and (h) SMAPE.

Fig 7 shows the performance of the proposed model for nature image set over conventional methods by varying noise levels from 0.02 to 0.08 . From the figure, it is detected that the PSNR of the proposed model is $65.14 \%$ and $16.53 \%$ better than 2D-SMCWT and DT-CWT, respectively. The SDME of the proposed model is $89.28 \%$ and $90 \%$ superior to 2D-SMCWT and DT-CDWT, respectively. Similarly, the same analysis is made for all measures, and the superiority of the proposed model is proved from the attained results.

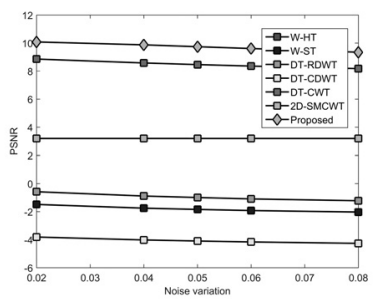

(a)

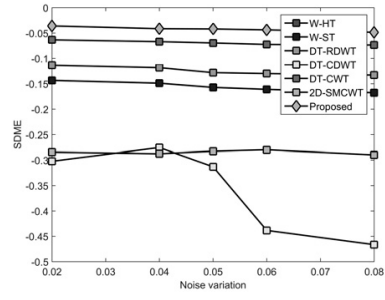

(b)

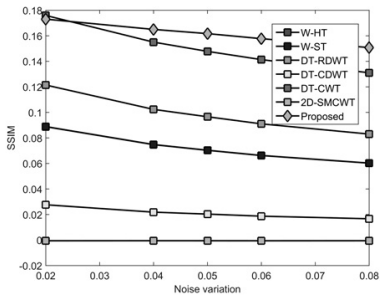

(c)

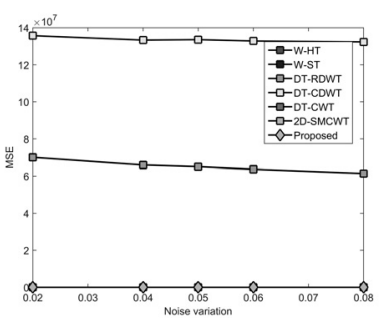

(d) 


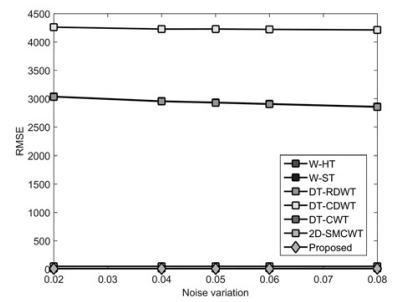

(e)

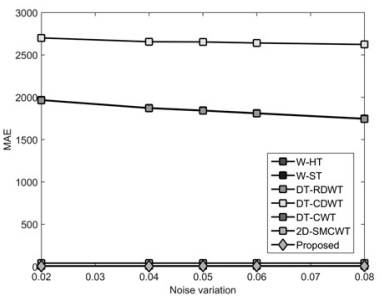

(f)

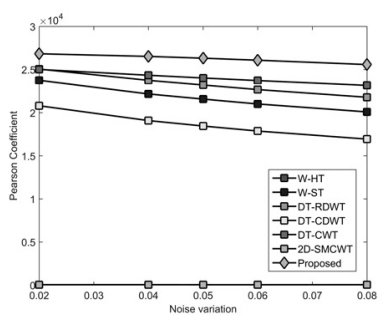

(g)

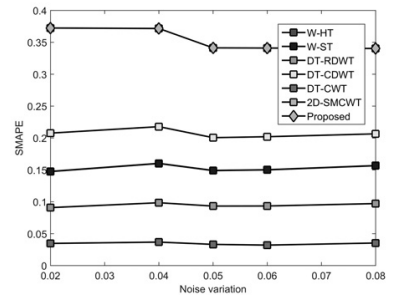

(h)

Fig. 7. Performance analysis of the proposed denoising model for Nature image set by varying noise in terms of (a) PSNR, (b) SDME, (c)SSIM, (d) MSE, (e) RMSE, (f) MAE, (g) PC, and (h) SMAPE.

Fig 8 illustrates the performance of the proposed model for medical image set over conventional methods by varying noise levels from 0.02 to 0.08 . It is evident that the PSNR of the proposed model is $65.82 \%, 9.96 \%, 58.78 \%$, $12.01 \%$, and $41.24 \%$ superior to 2D-SMCWT, DT-CWT, DT-CDWT, DT-RDWT, and W-ST, respectively. The SDME of the proposed model is $82.14 \%, 37.5 \%, 44.445$, and $58.3 \%$ better than 2D-SMCWT, DT-CWT, DT-RDWT, and W-ST, respectively.

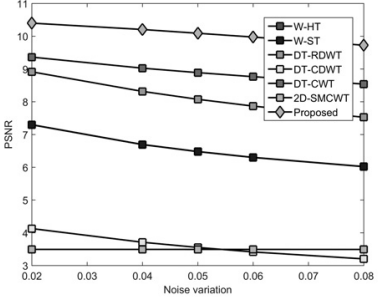

(a)

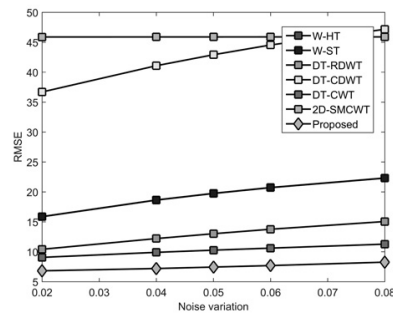

(e)

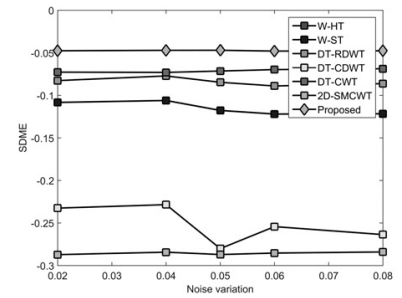

(b)

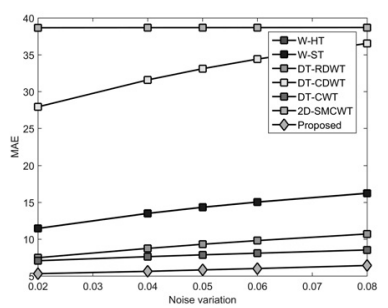

(f)

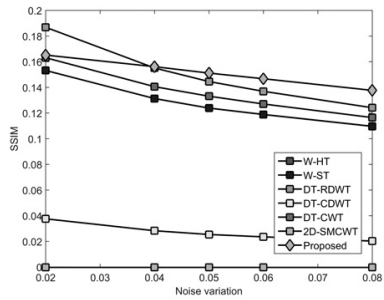

(c)

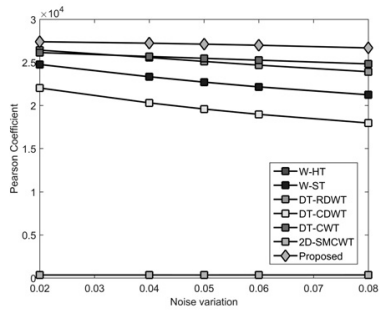

(g)

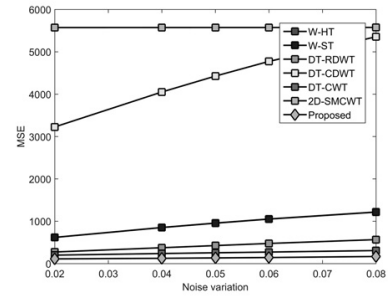

(d)

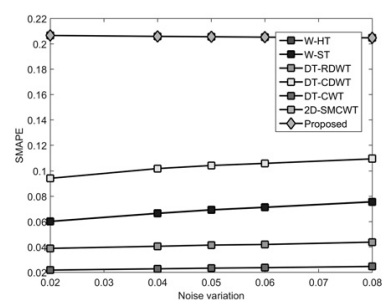

(h)

Fig. 8. Performance analysis of the proposed image denoising model for Medical image set by varying noise in terms of (a) PSNR, (b) SDME, (c)SSIM, (d) MSE, (e) RMSE, (f) MAE, (g) PC, and (h) SMAPE.

Fig 9 shows the performance of the proposed model for satellite image set over conventional methods by varying noise levels from 0.02 to 0.08 . It is observed that the PSNR of the proposed model is $61.93 \%, 34.095$, and $51.13 \%$ superior to 2D-SMCWT, DT-RDWT, and W-ST, respectively. Fig 10 shows the performance of the proposed model for miscellaneous image set over conventional methods by varying noise levels from 0.02 to 0.08 . From the figure, it is observed that the PSNR of the proposed model is $17.67 \%, 99.9 \%, 55.6 \%$, and $67.02 \%$ superior to DT-CWT, DT-CDWT, DT-RDWT, and W-ST, respectively. Similarly, the performance analysis of the proposed model for both satellite and miscellaneous image set is analyzed in terms of SDME, SSIM, MSE, RMSE, MAE, PC, and SMAPE, respectively. From the observed results, it is evident that the proposed model is superior to the conventional methods in terms of performance. 


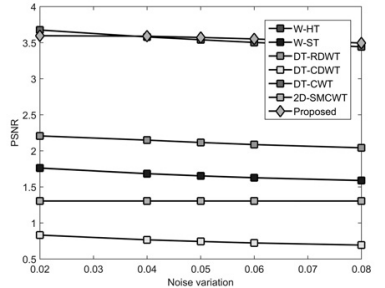

(a)

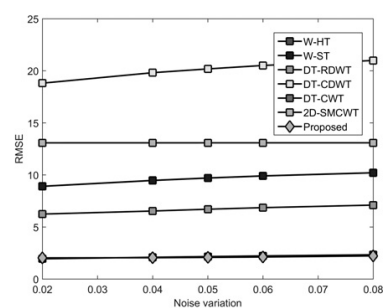

(e)

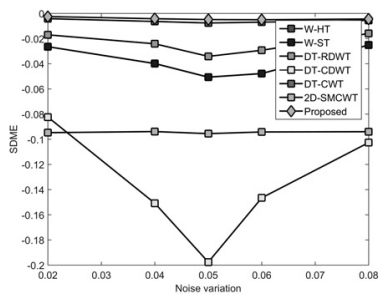

(b)

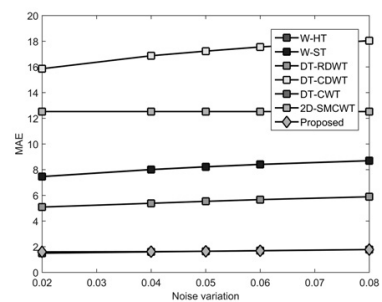

(f)

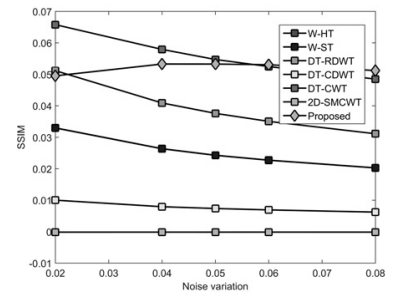

(c)

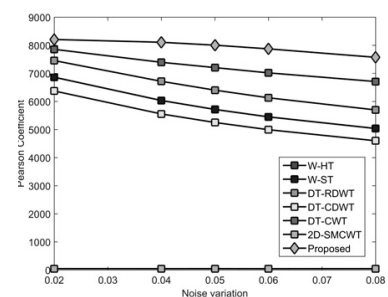

(g)

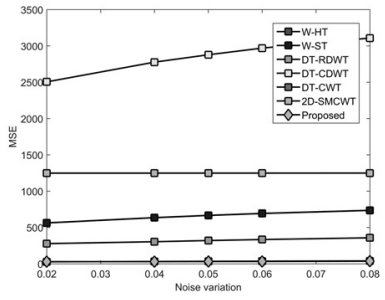

(d)

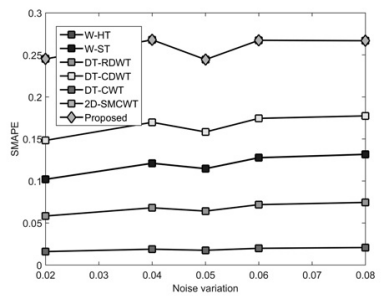

(h)

Fig. 9. Performance analysis of the proposed model for Satellite image set by varying noise in terms of (a) PSNR, (b) SDME, (c)SSIM, (d) MSE, (e) RMSE, (f) MAE, (g) PC, and (h) SMAPE.

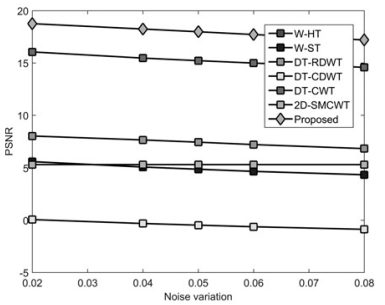

(a)

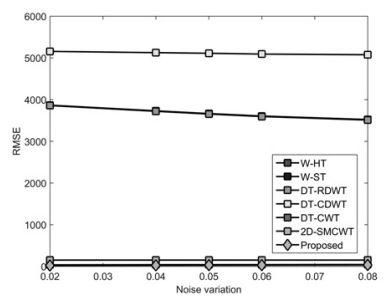

(e)

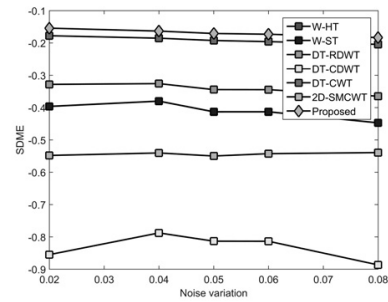

(b)

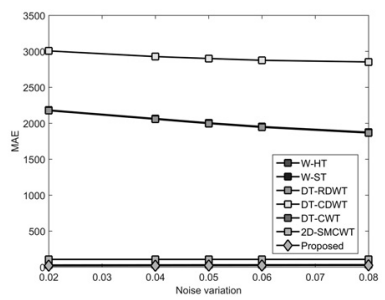

(f)

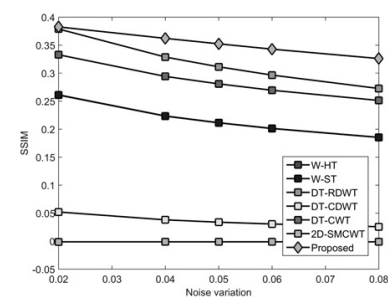

(c)

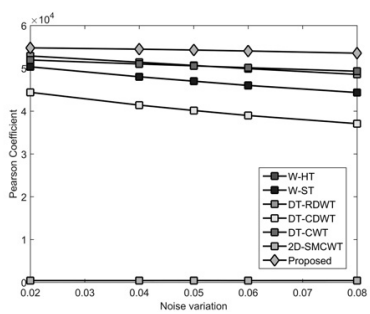

(g)

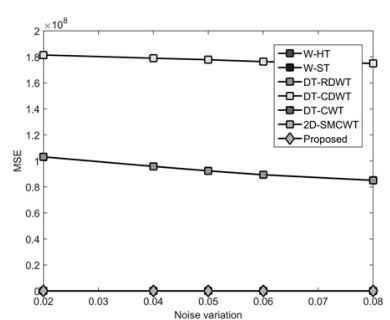

(d)

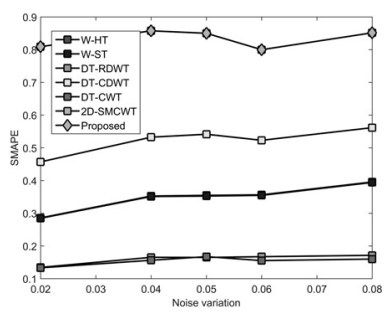

(h)

Fig. 10. Performance analysis of the proposed model for Miscilleneous image set by varying noise in terms of (a) PSNR, (b) SDME, (c)SSIM, (d) MSE, (e) RMSE, (f) MAE, (g) PC, and (h) SMAPE.

\section{Computing Complexity}

A model should be considered as an efficient model only if it incurs less computation complexity. Fig 11 shows the observed computational time of the proposed model. From Fig 11 it is detected that the proposed model falls on the second place with computing time $0.15 \mathrm{sec}$. Even though it is in the second place, while analyzing the performance of developed model, it is proved that the proposed model is more efficient than the other conventional methods. 


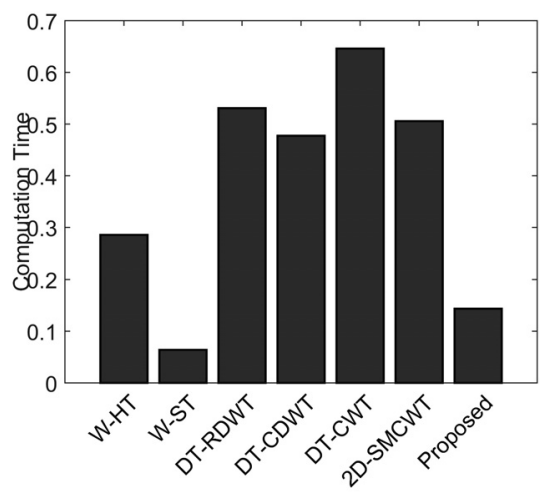

Fig. 11. Computational complexity of the proposed over conventional image denoising model.

\section{CONCLUSION}

This paper has developed a denoising algorithm for multi-modal images. Here, DT-CWT was exploited for image transformation for which the wavelet coefficients were estimated using BR classifier. The performance of denoising model was ensured by extracting the statistical and wavelet features. Consequently, the image characteristics were combined with noise spectrum for the development of BR model that has estimated the wavelet coefficients for effective denoising. Thus, the proposed denoising algorithm has exploited two stages of BR. The first stage has predicted the type of image, whereas the second stage has estimated optimal wavelet filter coefficients to DT-CWT for denoising. The performance of the proposed model was analyzed in terms of certain measures like PSNR, SDME, SSIM, MSE, RMSE, MAE, PC, and SMAPE, respectively, by comparing the developed model with other conventional models. From the results, it was observed that, for Texture image, the proposed model had attained promising results. The PSNR, SDME, SSIM, MSE, MAE, PC, and SMAPE of the developed model showed approximately $46.49 \%$, $85.79 \%, 54.72 \%, 80.62 \%, 59.33 \%, 29.75 \%$, and $68.25 \%$ superior to 2D SMCWT, DT-CWT, DT-CDWT, DT-RDWT, W-ST, and W-HT, respectively.

\section{REFERENCES}

Aggarwal, K.K., Singh, Y., Chandra, P. \& Puri, M. (2005). Bayesian Regularization in a Neural Network Model to Estimate Lines of Code Using Function Points, Journal of Computer Sciences, 1(4): 505-509.

Beck, A. \& Teboulle, M. (2009). A fast iterative shrinkage-thresholding algorithm for linear inverse problems, SIAM Journal of Imaging Sciences, 2(1): 183-202.

Call, J.M. (2005). Genetic algorithms for modelling and optimisation, Journal of Computational and Applied Mathematics, 184(1): 205-222.

Chung, H., Plourde, E. \& Champagne, B. (2017). Regularized non-negative matrix factorization with Gaussian mixtures and masking model for speech enhancement, Speech Communication, 87: 18-30.

Decker, A.D., Lee, J.A. \& Verleysen, M. (2010). A principled approach to image denoising with similarity kernels involving patches, Neurocomputing, 73(7-9):1199-1209.

Ghorai, S.K. (2013). A novel blind source separation technique using fractional Fourier transform for denoising medical images, Optik - International Journal for Light and Electron Optics, 124(3): 265-271.

Gong, M., Zhang, P., Su, L \& Liu, J. (2016). Cou-pled dictionary learning for change detection from multisourcedata," IEEE Transactions on Geoscience and Remote Sensing, 54(12): 7077-7091.

Guo, X., Li, Y., Suo, T. \& Liang, J. (2017). Denoising of digital image correlation based on stationary wavelet transform, Optics and Lasers in Engineering, 90: 161-172. 
Hanson, K.M. (1998). A simplified method of estimating noise power spectra, Physics of Medical Imaging, pp. $243-250$.

Hou, R., Wang, Z., Diamond, J.J., Zheng, Z., Zhu, J., Wang, Z. \& Chu, B. (2011). A quantitative evaluation model of denoising methods for surface plasmon resonance imaging signal, Sensors and Actuators B: Chemical, 160(1): 951-956.

Jesus, H., Domínguez, O., Máynez, L.O., Villegas, O.O.V., Mederos, B., Mejía, J.M. \& Sanchez, V.G.C. (2015). Denoising of high resolution small animal 3DPET data using the non-sub sampled Haar wavelet transform, Nuclear Instruments and Methods in Physics Research, 784: 581-584.

Jin, W.U. (2013). Wavelet domain denoising method based on multistage median filtering, The Journal of China Universities of Posts and Telecommunications, 20(2): 113-119.

Li, D., Zhang, L., Yang, J. \& Su, W. (2016). Research on wavelet-based contourlet transform algorithm for adaptive optics image denoising, Optik - International Journal for Light and Electron Optics, 127(12): 5029-5034.

Liu, C., Szeliski, R. \& Kang, S.B. (2008). Automatic estimation and removal of noise from a single image, IEEE Transactions on Pattern Analysis and Machine Intelligence, 30(2): 299-314.

Liu, T.C.K., Dong, X. \& Lu, W.S. (2009). Multiresolution wavelet denoising for ultra-wideband time-of-arrival estimation with regularized least squares, Physical Communication, 2(4): 285-295.

Liu, X. \& Fang, S. (2015). A convenient and robust edge detection method based on ant colony optimization, Optics Communications, 353: 147-157.

Liu, Z., Li, G., Mercier, G., He, Y \& Pan, Q. (2018). Change detection in heterogenous remote sensing im-ages via homogeneous pixel transformation," IEEE Trans-actions on Image Processing, 27(4): 1822-1834.

Liu, Z., Zhang, L., Li, G \& He, Y. (2017). Change de-tection in heterogeneous remote sensing images based on thefusion of pixel transformation," Information Fusion (Fu-sion), 2017 20th International Conference on. IEEE, 1-6.

Luisier, F., Blu, T. \& Unser, M. (2007). A new SURE approach to image denoising: Interscale orthonormal wavelet thresholding, IEEE Transactions on Image Processing, 16(3): 593-606.

Luo, J. \& Zhu, Y. (2012). Denoising of medical images using a reconstruction-average mechanism, Digital Signal Processing, 22(2): 337-347.

Luppino, L.T., Anfinsen, S.N., Moser, G., Jenssen, R., Bianchi, F.M., Ser-pico, S \& Mercier, G. (2017). A clustering approach to hetero-geneous change detection," in Scandinavian Conference onImage Analysis. Springer, 181-192.

Mercier, G., Moser, G \& Serpico. S.B. (2008). Conditional copulasfor change detection in heterogeneous remote sensing images,'IEEE Transactions on Geoscience and Remote Sensing, 46(5): 1428-1441.

Mohan, Y., Chee, S.S., Xin, D.K.P. \& Foong, L.P. (2016). Artificial neural network for classification of depressive and normal in EEG, 2016 IEEE EMBS Conference on Biomedical Engineering and Sciences (IECBES), Kuala Lumpur, pp. 286-290.

Naimi, H., Houda, A.B., Mitiche, A. \& Mitiche, L. (2015). Medical image denoising using dual- tree complex thresholding wavelet transform and Wiener filter, Journal of King Saud University - Computer and Information Sciences, 27(1): 40-45.

Nasri, M. \& Nezamabadi-pour, H. (2009). Image denoising in the wavelet domain using a new adaptive thresholding function, Neurocomputing, 72(4-6): 1012-1025.

Pizurica, A. \& Philips, W. (2006). Estimating the probability of the presence of a signal of interest in multiresolution single-and multiband image denoising, IEEE Transactions on Image Process, 15(3): 645-665.

Portilla, J., Strela, V., Wainwright, M.J. \& Simoncelli, E.P. (2003). Image denoising using scale mixtures of Gaussians in the wavelet domain, IEEE Transactions on Image Process, 12(11): 1338-1351.

Prendes, J., Chabert, M., Pascal, F., Giros, A \& Tourneret. J. (2015). A new multivariate statistical modelfor change detection in images acquired by homogeneous andheterogeneous sensors," IEEE Transactions on Image Process-ing, 24(3): 799-812.

Rabbani, H. (2009). Image denoising in steerable pyramid domain based on a local Laplace prior, Pattern Recognition, 42(9): 2181-2193.

Remenyi, N., Nicolis, O., Nason, G. \& Vidakovic, B. (2014). Image Denoising With 2D Scale-Mixing Complex Wavelet Transforms, IEEE Transactions on Image Processing, 23(12): 5165-5174. 
Sable, A.H \& Jondhale, K.C. (2010). Modified Double Bilateral Filter for Sharpness Enhancement and Noise Removal.

Selesnick, I.W., Baraniuk, R.G. \& Kingsbury, N.G. (2005). The Dual-Tree Complex Wavelet Transform, IEEE SIGNAL PROCESSING MAGAZINE, pp. 123-150.

Shang, L., Su, P. and Liu, T. (2012). Denoising MMW image using the combination method of contourlet and KSC shrinkage, Neurocomputing, 83: 229-233.

Song, C., Deng, H., , Gao, H., Zhang, H. \& Zuob, W. (2016). Bayesian non-parametric gradient histogram estimation for textureenhanced image deblurring, Neurocomputing, 197: 95-112.

Srivastava, R. (2014). Performance measurement of image processing algorithms. Professor, Department of Computer Science \& Engineering, IIT BHU, India,.

Storvik, B., Storvik, G \& Fjortoft, R. (2009). On the combi-nation of multisensor data using meta-gaussian distributions,"'IEEE Transactions on Geoscience and Remote Sensing, 47(7): 2372-2379.

Su, L., Gong, M., Zhang, P., Zhang, M., Liu, J \& Yang, H. (2017). Deep learning and mapping based ternary change detection for information unbalanced images,"Pattern Recognition, 66: 213-228.

Sun, D., Gao, Q., Lu, Y., Huang, Z. \& Li, T. (2014). A novel image denoising algorithm using linear Bayesian MAP estimation based on sparse representation, Signal Processing, 100: 132-145.

Sunil, B.S., Manjunath, A.S., Christopher, S. \& Menon, R.L. (2016). Motion-compensated adaptive dual tree complex wavelet transform coding for scalable color video compression using SPIHT, 9: 47-57.

SunilKumar, B.S., Manjunath, A.S., Christopher, S. \& Menon, R. (2016). Enhanced Scalable Video Coding Technique with an Adaptive Dual- Tree Complex Wavelet Transform, Procedia Computer Science, 85: 70-77.

Touati, R \& Mignotte, M. (2018). An energy-based modelencoding nonlocal pairwise pixel interactions for multisensorchange detection," IEEE Transactions on Geoscience and Re-mote Sensing, 56(2): 1046-1058.

Tuia, D., Marcos \& G., Camps-Valls. (2016). Multi-temporal and multi-source remote sensing image classificationby nonlinear relative normalization,” ISPRS Journal of Pho-togrammetry and Remote Sensing, 120: 1-12.

Volpi, M., Camps-Valls, G \& Tuia, D. (2015). Spec-tral alignment of multi-temporal cross-sensor images with au-tomated kernel canonical correlation analysis," ISPRS Journalof Photogrammetry and Remote Sensing, 107: 50-63.

Wagh \& Todmal. (2015). Eyelids, Eyelashes Detection Algorithm and Hough Transform Method for Noise Removal in Iris Recognition", International Journal of Computer Applications, 112(3).

Wang, X.Y. \& Kai Fu, Z. (2010). A wavelet-based image denoising using least squares support vector machine, Engineering Applications of Artificial Intelligence, 23(6): 862-871.

Wang, X.Y., Yang, H.Y. \& Kai Fu, Z. (2010). A New Wavelet-based image denoising using undecimated discrete wavelet transform and least squares support vector machine, Expert Systems with Applications, 37: 7040-7049.

Wang, X.Y., Yang, H.Y., Zhang, Y. \& Kai Fu, Z. (2013). Image denoising using SVM classification in nonsubsampled contourlet transform domain, Information Sciences, 246: 155-176.

Wong, A., Mishra, A., Zhang, W., Fieguth, P. \& Clausi, D.A. (2011). Stochastic image denoising based on Markov-chain Monte Carlo sampling, Signal Processing, 91(8): 2112-2120.

Xin, Z. \& Jiangtao, Q. (2011). De-noising of GIS UHF Partial Discharge Monitoring based on Wavelet Method, Procedia Environmental Sciences, 11: 1302-1307.

Yan, F., Cheng, L. \& Peng, S. (2008). A new interscale and intrascale orthonormal wavelet thresholding for SURE-based image denoising, IEEE Transactions on Signal Processing Letters, 15: 139-142.

Yang, H.Y., Wang, X.Y. \& Kai Fu, Z. (2012). A new image denoising scheme using support vector machine classification in shiftable complex directional pyramid domain, Applied Soft Computing, 12(2): 872-886.

YueSi, Zhang, Z., Wang, H. \& Yuan, F. (2016). Mono modal feature extraction for bonding quality detection of explosive clad structure with optimized dual-tree complex wavelet transform, Journal of Sound and Vibration.

Zhang, P., Gong, M., Su, L., Liu, J \& Li, Z. (2016). Change detection based on deep feature representation and mapping 
transformation for multi-spatial-resolution re-mote sensing images," ISPRS Journal of Photogrammetry and Remote Sensing, 116: $24-41$.

Zhang, W. (2013). Image denoising algorithm of refuge chamber by combining wavelet transform and bilateral filtering, International Journal of Mining Science and Technology, 23(2): 221-225.

Zhao, W., Wang, Z., Gong, M \& Liu, J. (2017). Discriminative feature learning for unsupervised change detectionin heterogeneous images based on a coupled neural network,"IEEE Transactions on Geoscience and Remote Sensing.

Submitted: 28/12/2017

Revised: $\quad 01 / 05 / 2019$

Accepted: $02 / 05 / 2019$ 


\title{
استخدام شبكة بايزي ثنائية المرحلة مع التحويل المويجي المعقد ذي الشجرة الثنائية لتقليل تشويش الصور
}

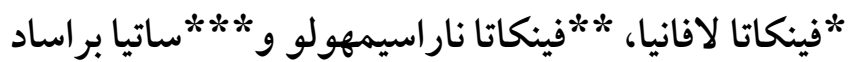

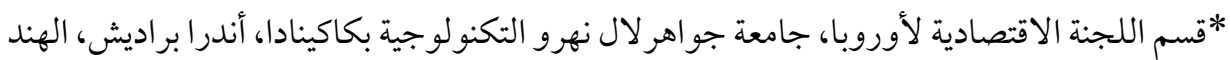

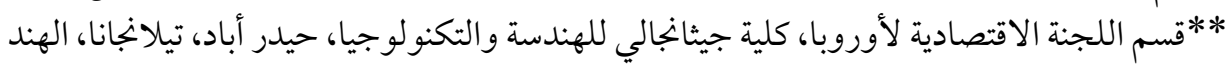

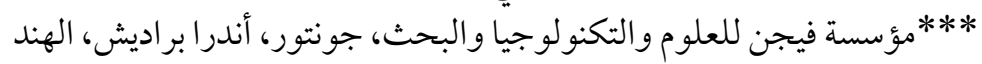

\section{الخالاصة}

إزالة التشويش من الصور تلعب دوراً حيوياً في العروض الهندسية المختلفة. وكذلك في تكنولوجيا معالجة الصور، ظل الخلاف

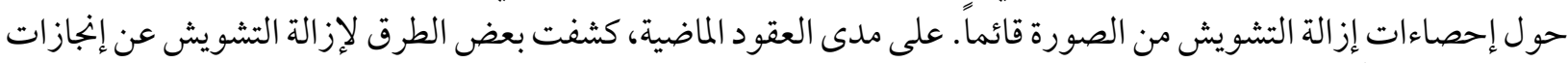

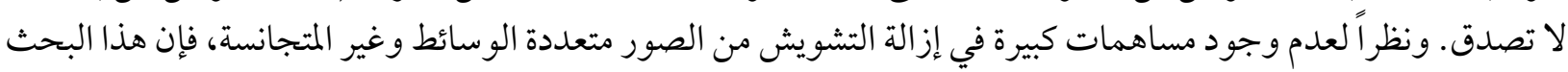

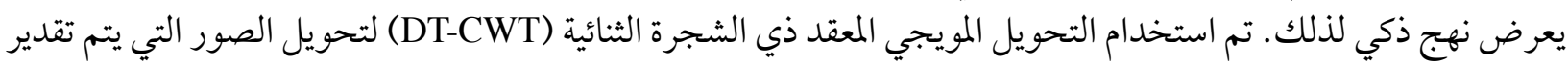

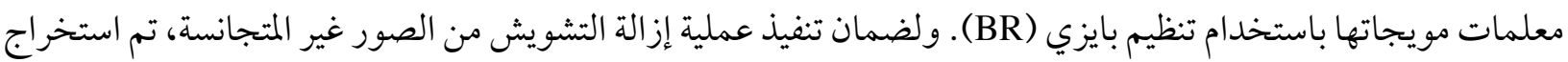

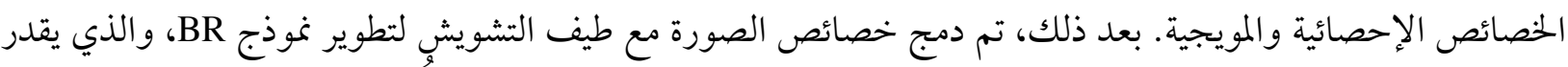

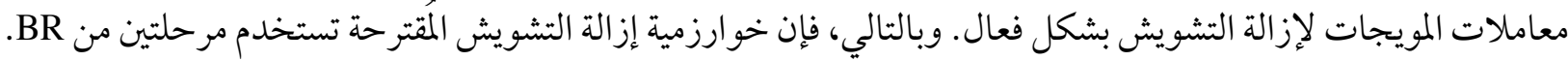

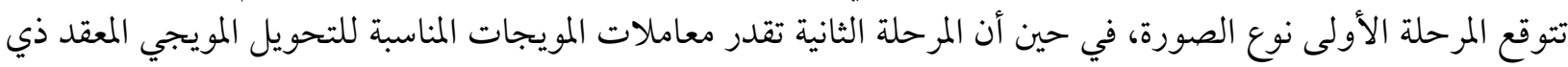

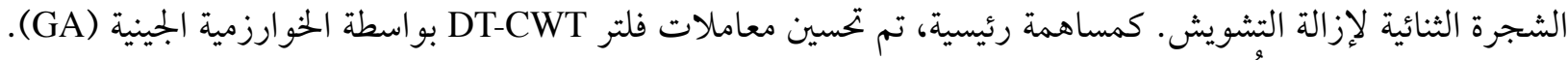

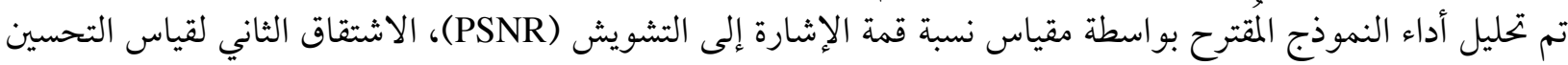

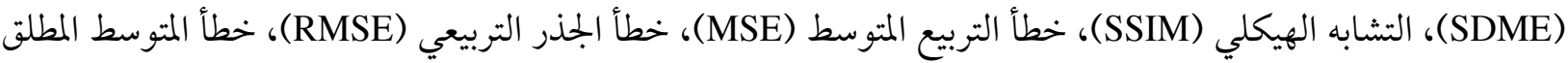

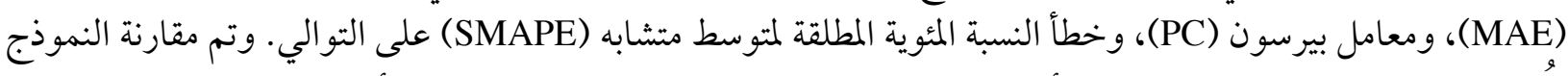

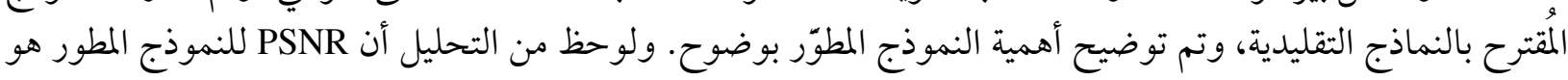

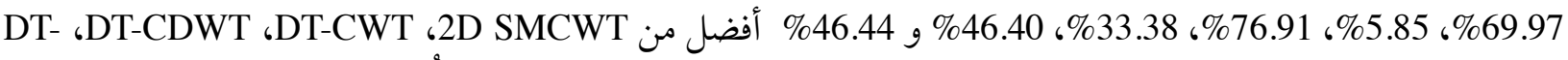

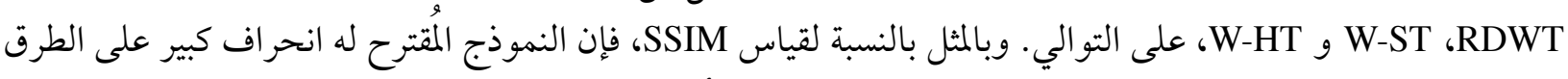

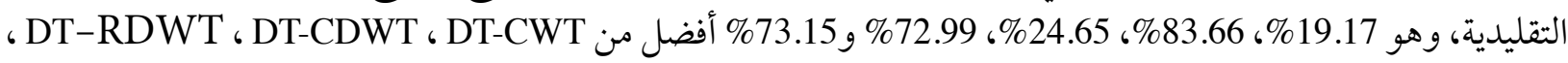
و و ST- ST 